\title{
Boundary Layer Effects on Liners for Aircraft Engines
}

\author{
Gwénaël Gabard \\ Institute of Sound and Vibration Research, University of Southampton, UK
}

\begin{abstract}
The performance of acoustic treatments installed on aircraft engines is strongly influenced by the boundary layer of the grazing flow on the surface of the liner. The parametric study presented in this paper illustrates the extent of this effect and identifies when it is significant. The acoustic modes of a circular duct with flow are calculated using a finite difference method. The parameters are representative of the flow conditions, liners and sound fields found in current turbofan engines. Both the intake and bypass ducts are considered. Results show that there is a complex interplay between the boundary layer thickness, the direction of propagation and the liner impedance and that the boundary layer can have a strong impact on liner performance for typical configurations (including changes of the order of $30 \mathrm{~dB}$ on the attenuation of modes associated with tonal fan noise). A modified impedance condition including the effect of a small but finite boundary layer thickness is considered and compared to the standard Myers condition based on an infinitely thin boundary layer. We show how this impedance condition can be implemented in a mode calculation method by introducing auxiliary variables. This condition is able to capture the trends associated with the boundary layer effects and in most cases provides improved predictions of liner performance.
\end{abstract}

Keywords: Impedance; liner; Myers; grazing flow; boundary layer; turbofan

\section{Introduction}

The challenge when predicting sound absorption by acoustic treatments in the presence of grazing flow is to describe the interactions between the sound field, the boundary layer over the surface, and the liner. A complete description of a typical honeycomb-perforate liner would include the micro-fluid dynamics near the holes on the facing sheet, the sound field in the honeycomb cavities, the mean flow and the turbulent structures in the boundary layer and the incoming sound field interacting with all of these. For practical purposes most of these aspects are heavily simplified. However, from an engineering point of view there is still a need for improving the attenuation achieved by turbofan liners to further reduce noise emission from air transport (this is also relevant to applications in the automotive industry). In addition it is important to reduce the uncertainty associated with existing models based on a simplified description of the boundary layer.

The model commonly used for this purpose is the Myers condition [1] where the interaction between sound and the mean boundary layer is described by a vortex sheet model, and the liner is characterised solely by its acoustic impedance. There are however a number of shortcomings associated with such a simple model. It is not well-posed in the time domain, which causes various difficulties for computational simulations $[2,3]$. The actual behaviour of the hydrodynamic field above the liner is quite different from what is predicted by the Myers condition since the vortex sheet is a crude description of a compressible boundary layer $[4,5,6]$. Several published results indicated that the boundary layer has a significant effect on liner attenuation $[7,8,9]$, but most of these results are either experimental data for small ducts where the flow velocity and boundary layer are not always representative of the actual intake ducts for instance [10], or theoretical predictions for specific modes or conditions relatively different from turbofan engine liners. The impact of the boundary layer refraction on the performance of a liner was studied by Rice $[11,12,13,14]$ who obtained correlation equations for the optimum impedance, the maximum attenuation and the cut-off ratio of the duct modes. These equations capture trends observed in the experimental data available at the 
time and show a significant impact of the boundary layer on the optimum impedance (see for instance figure 3 in [11]). However they rely on a number of assumptions and simplifications.

More recently, several modified impedance conditions have been proposed to provide improved predictions $[15,16,4,17]$. The introduction of the boundary layer thickness as an additional parameter offers the potential for more accurate predictions of the performance of liners under grazing flow. A preliminary study [18] indicated that the effect of the boundary layer is not always well predicted by the Myers condition, and that the impedance condition proposed by Brambley [17] tends to provide a better description of this effect. However, the analysis in [18] was only considering a plane wave reflected off a flat surface.

The present paper reports a detailed parametric study of the boundary layer effects with a test case and parameters representative of the inlet and bypass ducts of current large turbofan engines. The aim is to clarify in which situations this effects is significant and what are the parameters that are important in this context. This is done by calculating the attenuation rate of a large number of duct modes in various conditions of flow liner. Several models for the boundary layer are compared (see section 2): (i) uniform flow with the Myers condition, (ii) uniform flow with the modified impedance condition proposed in [17] and (iii) a reference solution with the actual boundary layer profile with the no-flow impedance condition at the wall. A high-order finite difference model is used to calculate the acoustic modes (see section 3). A range of parameters is considered to cover the different flow conditions, sound fields and liners found in the intake and bypass ducts of a modern turbofan engine. In the discussion of the results (sections 5 to 7 ) we attempt to address the following issues: (i) Provide a quantitative assessment of the effect of the boundary layer and identify in which cases it is significant, and what are the modes sensitive to its influence; (ii) Identify when the Myers impedance condition is unable to capture the influence of the boundary layer; (iii) Assess the modified impedance condition to determine if and when it can provide improved predictions. The main conclusions are then summarised in section 8 .

\section{Governing Equations}

We consider a straight duct with circular cross section and radius $R$. The duct axis is $z$, the radial and azimuthal coordinates are $r$ and $\theta$. The duct carries an axi-symmetric, axial flow with velocity $u_{0}(r)$, mean density $\rho_{0}(r)$ and sound speed $c_{0}(r)$. Viscosity and heat conduction are neglected.

\subsection{Linearised Euler equations}

The propagation of small perturbations of the mean flow is described by the linearised Euler equations, written here in cylindrical coordinates:

$$
\frac{\partial \mathbf{q}}{\partial t}+\frac{1}{r} \frac{\partial}{\partial r}\left(r \mathbf{F}_{r} \mathbf{q}\right)+\frac{1}{r} \frac{\partial}{\partial \theta}\left(\mathbf{F}_{\theta} \mathbf{q}\right)+\frac{\partial}{\partial z}\left(\mathbf{F}_{z} \mathbf{q}\right)+\frac{1}{r} \mathbf{F}_{c} \mathbf{q}=\mathbf{0}
$$

where we have introduced the vector

$$
\mathbf{q}=\left[\begin{array}{c}
\rho^{\prime} \\
\left(\rho u_{z}\right)^{\prime} \\
\left(\rho u_{r}\right)^{\prime} \\
\left(\rho u_{\theta}\right)^{\prime} \\
\hat{p}^{\prime}
\end{array}\right]
$$

which contains the perturbations of density $\rho^{\prime}$, momentum $(\rho \mathbf{u})^{\prime}$ and modified pressure variable $\hat{p}^{\prime}$ defined as $\hat{p}=\left(p / p_{\infty}\right)^{1 / \gamma}$ where $\gamma$ is the ratio of specific heats and $p_{\infty}$ is a reference value for pressure. It follows that the perturbation of $\hat{p}$ is simply related to the acoustic pressure by $p^{\prime}=\rho_{0} c_{0}^{2} \hat{p}^{\prime} / \hat{p}_{0}$. This modified variable is related to that introduced by Goldstein for an acoustic analogy in parallel sheared flows [19, eq. 3.7]. The use of this modified variable is preferred here as it allows to write the Euler equations in a conservative form, thus avoiding the presence of mean flow gradients in the governing equations. The matrices $\mathbf{F}_{z}, \mathbf{F}_{r}$ and $\mathbf{F}_{\theta}$ represent the fluxes in each direction, and the matrix $\mathbf{F}_{c}$ describes the Coriolis forces due to the use 
of cylindrical coordinates. The definitions of these matrices are as follows:

$$
\begin{gathered}
\mathbf{F}_{z}=\left[\begin{array}{ccccc}
0 & 1 & 0 & 0 & 0 \\
-u_{0}^{2} & 2 u_{0} & 0 & 0 & \rho_{0} c_{0}^{2} / \hat{p}_{0} \\
0 & 0 & u_{0} & 0 & 0 \\
0 & 0 & 0 & u_{0} & 0 \\
-u_{0} \hat{p}_{0} / \rho_{0} & \hat{p}_{0} / \rho_{0} & 0 & 0 & u_{0}
\end{array}\right], \quad \mathbf{F}_{r}=\left[\begin{array}{ccccc}
0 & 0 & 1 & 0 & 0 \\
0 & 0 & u_{0} & 0 & 0 \\
0 & 0 & 0 & 0 & \rho_{0} c_{0}^{2} / \hat{p}_{0} \\
0 & 0 & 0 & 0 & 0 \\
0 & 0 & \hat{p}_{0} / \rho_{0} & 0 & 0
\end{array}\right], \\
\mathbf{F}_{\theta}=\left[\begin{array}{ccccc}
0 & 0 & 0 & 1 & 0 \\
0 & 0 & 0 & u_{0} & 0 \\
0 & 0 & 0 & 0 & 0 \\
0 & 0 & 0 & 0 & \rho_{0} c_{0}^{2} / \hat{p}_{0} \\
0 & 0 & 0 & \hat{p}_{0} / \rho_{0} & 0
\end{array}\right], \quad \mathbf{F}_{c}=\left[\begin{array}{ccccc}
0 & 0 & 0 & 0 & 0 \\
0 & 0 & 0 & 0 & 0 \\
0 & 0 & 0 & 0 & -\rho_{0} c_{0}^{2} / \hat{p}_{0} \\
0 & 0 & 0 & 0 & 0 \\
0 & 0 & 0 & 0 & 0
\end{array}\right] .
\end{gathered}
$$

We seek normal modes of the sound field in the duct by requiring that

$$
\mathbf{q} \sim \mathrm{e}^{\mathrm{i} \omega t-\mathrm{i} m \theta-\mathrm{i} k z},
$$

where $\omega$ is the angular frequency, $m$ is the azimuthal order of the mode and $k$ is the axial wavenumber. The governing equation (1) become

$$
\mathrm{i} \omega \mathbf{q}-\mathrm{i} k \mathbf{F}_{z} \mathbf{q}-\frac{\mathrm{i} m}{r} \mathbf{F}_{\theta} \mathbf{q}+\frac{1}{r} \mathbf{F}_{c} \mathbf{q}+\frac{1}{r} \frac{\partial}{\partial r}\left(r \mathbf{F}_{r} \mathbf{q}\right)=\mathbf{0} .
$$

For a prescribed frequency $\omega$ equation (2) represents a generalised eigenvalue problem for $k$ with eigenvector q. If the axial wavenumber is complex, the absorption rate of this mode is given by $\pm 20 \operatorname{Im}(k) / \ln (10)$ (in $\mathrm{dB} / \mathrm{m}$ ) where the + and - signs are used for mode propagating in the negative or positive directions, respectively.

\subsection{Fully-resolved boundary layer}

To describe the effect of the boundary layer on liner attenuation we consider that the flow is uniform except for a boundary layer of finite thickness $\delta$ at the wall. Since there is no mean flow at the wall we can directly use the standard impedance relation for a locally reacting liner characterised by its impedance $Z(\omega)$ :

$$
u_{r}^{\prime}=\frac{p^{\prime}}{Z(\omega)}, \quad \text { at } r=R .
$$

Fully resolving the boundary layer is more costly but will provide the reference solutions to assess the impedance conditions described in the next two sections.

\subsection{The Myers condition}

The first model we consider is that of an infinitely thin boundary layer above the liner $(k \delta \ll 1)$. This is described by the Ingard-Myers condition [20,1] where the boundary layer is represented by a vortex sheet across which the continuity of pressure and normal displacement is imposed [21]. For a flat surface this boundary condition reads

$$
\mathrm{i} \omega u_{r}^{\prime}=\mathrm{i}\left(\omega-k u_{0}\right) \frac{p^{\prime}}{Z(\omega)}, \quad \text { at } r=R .
$$

While, in this paper, we refer to equation (4) as an impedance condition, it is important to note that it is a combination of the standard impedance relation (3) with a simplified model for the transmission of sound through the boundary layer. It is applied directly at the wall and the mean flow is assumed uniform with a non-zero wall slip velocity $u_{0}$. The effect of the boundary layer is therefore not described by the governing equations (1) but is included in the Ingard-Myers condition. If there is no mean flow this condition reduces to the standard impedance relation (3). 
To be used with the eigenvalue problem (2), equation (4) is written

$$
\mathrm{i} \omega u_{r}^{\prime}-\mathrm{i} \omega \frac{p^{\prime}}{Z(\omega)}+\mathrm{i} k u_{0} \frac{p^{\prime}}{Z(\omega)}=0
$$

\subsection{Boundary condition with a finite boundary layer thickness}

A modified impedance condition has been proposed by Brambley [17] where the boundary layer thickness $\delta$ is taken to be small but finite. Outside the boundary layer the mean flow is uniform. Following the approach originally used by Eversman and Beckemeyer [21], this impedance condition is obtained by a matched asymptotic expansion of the Pridmore-Brown equation in the boundary layer. With our notations this boundary condition reads:

$$
u_{r}^{\prime}+\frac{k^{2}+m^{2} / R^{2}}{\mathrm{i} \rho_{0}(0)\left[\omega-k u_{0}(0)\right]} \delta I_{1} p^{\prime}=\frac{\omega-k u_{0}(0)}{\omega} \frac{p^{\prime}+\mathrm{i} \rho_{0}(0)\left[\omega-k u_{0}(0)\right] \delta I_{0} u_{r}^{\prime}}{Z(\omega)}, \quad \text { at } r=R,
$$

with

$$
\delta I_{0}=\int_{R-\delta}^{R} 1-\frac{\left[\omega-k u_{0}(r)\right]^{2} \rho_{0}(r)}{\left[\omega-k u_{0}(0)\right]^{2} \rho_{0}(0)} \mathrm{d} r, \quad \delta I_{1}=\int_{R-\delta}^{R} 1-\frac{\left[\omega-k u_{0}(0)\right]^{2} \rho_{0}(0)}{\left[\omega-k u_{0}(r)\right]^{2} \rho_{0}(r)} \mathrm{d} r .
$$

We use $u_{0}(0)$ and $\rho_{0}(0)$ to refer to the mean flow properties outside the boundary layer. By developing the integral for $\delta I_{0}$ it is possible to write:

$$
\delta I_{0}=\frac{1}{\left[\omega-k u_{0}(0)\right]^{2}}\left[\omega^{2} \delta_{0}-2 \omega k u_{0}(0) \delta_{1}+k^{2} u_{0}(0)^{2}\left(\delta_{1}+\delta_{2}\right)\right],
$$

where we have introduced

$$
\delta_{0}=\int_{R-\delta}^{R} 1-\frac{\rho_{0}(r)}{\rho_{0}(0)} \mathrm{d} r, \quad \delta_{1}=\int_{R-\delta}^{R} 1-\frac{\rho_{0}(r) u_{0}(r)}{\rho_{0}(0) u_{0}(0)} \mathrm{d} r, \quad \delta_{2}=\int_{R-\delta}^{R} \frac{\rho_{0}(r) u_{0}(r)}{\rho_{0}(0) u_{0}(0)}\left[1-\frac{u_{0}(r)}{u_{0}(0)}\right] \mathrm{d} r
$$

$\delta_{1}$ is the displacement thickness of the boundary layer and $\delta_{2}$ is the momentum thickness. $\delta_{0}$ can be considered as the 'mass' thickness as it is only associated with the mean density profile. Its effect will not be considered here, since only a uniform density profile will be used. Note that the general definitions for the displacement and momentum thicknesses of a boundary layer in a circular duct are more complicated, but the expressions in the equation above correspond to the approximations of these definitions to first order in $\delta$.

The $\delta I_{1}$ term cannot be simplified in the same way as $\delta I_{0}$. One exception, which we will use here, is that of a boundary layer with a linear velocity profile and a constant mean density. In this case we have:

$$
\delta I_{1}=\frac{u_{0}(0) k \delta}{\omega}, \quad \delta_{0}=0, \quad \delta_{1}=\frac{1}{2} \delta, \quad \delta_{2}=\frac{1}{6} \delta
$$

and equation (6) becomes:

$$
\mathrm{i} \omega \rho_{0}(0) u_{r}^{\prime}+\mathrm{i} k \delta u_{0} \frac{k^{2}+m^{2} / R^{2}}{\mathrm{i} \omega-\mathrm{i} k u_{0}(0)} p^{\prime}=\mathrm{i} \rho_{0}(0)\left[\omega-k u_{0}(0)\right] \frac{p^{\prime}}{Z(\omega)}+\rho_{0}(0) u_{0}(0) k \delta\left[\omega-\frac{2}{3} k u_{0}(0)\right] \frac{\rho_{0}(0) u_{r}^{\prime}}{Z(\omega)} .
$$

As for the Myers condition (4), equation (8) is applied directly at the wall $r=R$ with a uniform flow instead of the boundary layer. In other words, the impedance condition (8) is an approximation of the equivalent boundary condition required in the uniform flow case in order to recover the same results as with a boundary layer.

One remaining issue is that equation (8) is not in a form suitable for the eigenvalue problem (2) where all the terms should be independent of $k$ or linear in $k$. This is addressed by first rewriting the second term on the left-hand side in terms of the axial and azimuthal components of velocity. Since we are assuming a 
uniform flow above the impedance condition these components are related to pressure by

$$
\mathrm{i}\left[\omega-k u_{0}(0)\right] \rho_{0}(0) u_{z}^{\prime}-\mathrm{i} k p^{\prime}=0, \quad \text { and } \quad \mathrm{i}\left[\omega-k u_{0}(0)\right] \rho_{0}(0) u_{\theta}^{\prime}-\mathrm{i} \frac{m}{R} p^{\prime}=0 .
$$

The second term on the left-hand side in (8) can therefore be written

$$
\mathrm{i} k \delta u_{0} \frac{k^{2}+m^{2} / R^{2}}{\mathrm{i} \omega-\mathrm{i} k u_{0}(0)} p^{\prime}=-\mathrm{i} k \delta u_{0}\left[\mathrm{i} k \rho_{0}(0) u_{z}^{\prime}+\mathrm{i} \frac{m}{R} \rho_{0}(0) u_{\theta}^{\prime}\right] .
$$

To obtain a set of equations with only linear terms in $k$ we have to introduce two auxilliary variables that are defined only on the boundary:

$$
\alpha^{\prime}=\rho_{0}(0)\left(\mathrm{i} k u_{z}^{\prime}+\mathrm{i} \frac{m}{R} u_{\theta}^{\prime}\right), \quad \beta^{\prime}=\mathrm{i}\left(\omega-k \frac{2}{3} u_{0}\right) u_{r}^{\prime} .
$$

The impedance boundary condition can finally be written

$$
\mathrm{i} \omega\left(\rho u_{r}\right)^{\prime}-\mathrm{i} k \delta u_{0} \alpha^{\prime}-\mathrm{i} \rho_{0}\left(\omega-k u_{0}\right) \frac{p^{\prime}}{Z(\omega)}+\mathrm{i} k \delta \rho_{0} u_{0} \frac{\beta^{\prime}}{Z(\omega)}=0 .
$$

The additional variables $\alpha^{\prime}$ and $\beta^{\prime}$ are only defined on the boundary. The restriction to a linear boundary layer profile is required here in order to write the boundary condition in a way that is compatible with the eigenvalue problem (2). But this is not necessarily restrictive since it was observed in [18] and [8] that the actual velocity profile of the boundary layer only has a small impact on the liner attenuation provided that the displacement thickness $\delta_{1}$ is kept constant.

\section{Numerical Solutions}

\subsection{Finite-difference approximations}

The eigenvalue problem (2) is solved numerically using a high-order finite-difference method. The grid points are distributed between $r=0$ and $r=R$ and denoted $r_{n}$ with $n=1, \ldots, N$. Seven-point, fourth-order DRP stencils $[22,23]$ are used to approximate the radial derivative in equation (2). This leads to an algebraic system of equations of the form:

$$
\mathrm{i} \omega \mathbf{Q}-\mathrm{i} k \mathbf{A}_{z} \mathbf{Q}-\mathrm{i} m \mathbf{A}_{\theta} \mathbf{Q}+\mathbf{A}_{r} \mathbf{Q}=\mathbf{0} .
$$

The vector $\mathbf{Q}$ contains all the unknowns $\mathbf{q}$ at the $N$ grid points, together with the auxiliary variables $\alpha^{\prime}$ and $\beta^{\prime}$ if the modified impedance condition (11) is used.

For a given frequency we solve this generalised eigenvalue problem for the eigenvalue $k$ and mode shape function $\mathbf{Q}$ using the Matlab function EIG based on the iterative QZ algorithm.

\subsection{Boundary conditions}

The discrete form (12) of the eigenvalue problem is modified to account for the boundary condition at the duct wall. All three impedance conditions (3), (4) and (6) specify the radial velocity. Each of these conditions is therefore used instead of the equation from (2) for the radial component of momentum. For the modified impedance condition (6), the two additional variables $\alpha^{\prime}$ and $\beta^{\prime}$ are introduced at the last grid point $r_{N}$. The additional equations for these unknowns are given by (10).

For the numerical treatment of the axis of symmetry, we use a technique similar to that of Mohseni \& Colonius [24] where additional grid points are added with negative radial coordinates to extend the grid beyond the axis of symmetry. Since we are using a seven-point stencil, three grid points $r_{-2}, r_{-1}$ and $r_{0}$ are added. They are mirror images of the first three grid points and are therefore defined by $r_{-k}=-r_{1+k}$ with $k=0,1,2$. The key is to ensure that $r_{1} \neq 0$ so that the apparent singularity in the governing equations (2) is not present at any grid point. 
The solutions at the additional grid points are not calculated with (2) but are defined directly by the $\mathrm{e}^{-\mathrm{i} m \theta}$ dependence for each aximuthal mode $m$. For a scalar field $f$ (i.e. either $\hat{p}^{\prime}$ or $\rho^{\prime}$ ) this yields:

$$
\rho^{\prime}(-r, \theta)=\rho^{\prime}(r, \theta) \mathrm{e}^{-\mathrm{i} m \pi}, \quad \hat{p}^{\prime}(-r, \theta)=\hat{p}^{\prime}(r, \theta) \mathrm{e}^{-\mathrm{i} m \pi} .
$$

For a vector field such as the acoustic velocity the conditions are

$$
u_{z}^{\prime}(-r, \theta)=+u_{z}^{\prime}(r, \theta) \mathrm{e}^{-\mathrm{i} m \pi}, \quad u_{r}^{\prime}(-r, \theta)=-u_{r}^{\prime}(r, \theta) \mathrm{e}^{-\mathrm{i} m \pi}, \quad u_{\theta}^{\prime}(-r, \theta)=-u_{\theta}^{\prime}(r, \theta) \mathrm{e}^{-\mathrm{i} m \pi} .
$$

The change in sign stems from the fact that the basis vectors $\mathbf{e}_{r}$ and $\mathbf{e}_{\theta}$ vary with $\theta$ whereas $\mathbf{e}_{z}$ is independent of position. Equations (13) and (14) are sufficient to fully specify the solutions at the three additional grid points.

Boundary conditions for symmetry axes are generally formulated in terms of parity conditions. Boyd provides a dicsussion of the use of this condition [25] and details of the derivation can be found in [26]. For cylindrical coordinates these conditions depend on the azimuthal order $m$ :

$$
\begin{aligned}
& \text { for } m=0: \quad u_{r}^{\prime}=0, \quad u_{\theta}^{\prime}=0, \\
& \text { for } m= \pm 1: \quad p^{\prime}=0, \quad \rho^{\prime}=0, \quad u_{z}^{\prime}=0, \quad u_{r}^{\prime}= \pm \mathrm{i} u_{\theta}^{\prime} \text {, } \\
& \text { for }|m|>1: \quad p^{\prime}=0, \quad \rho^{\prime}=0, \quad \mathbf{u}^{\prime}=\mathbf{0} \text {. }
\end{aligned}
$$

We have checked that the use of (13) and (14) yield solutions that satisfy the parity conditions above.

\subsection{Identification of well-resolved numerical modes}

Of all the eigenvalues and eigenvectors obtained from (12), only a small number can be considered to be sufficiently well resolved to correspond to physical modes. The other solutions are dominated by point-topoint oscillations and are not representative of the physics. It is therefore necessary to identify the modes that can be considered physical and discard the others.

The method used here for this purpose is based on selective filters developed for high-order finitedifference schemes. These filters are designed to retain only the very short wavelength components in a finite-difference solution. The filtered solution $\tilde{\mathbf{q}}$ is calculated as follows:

$$
\tilde{\mathbf{q}}\left(r_{n}\right)=\sum_{i=-3}^{+3} a_{i} \mathbf{q}\left(r_{n+i}\right)
$$

where $a_{i}$ are the coefficients of the filter (with $a_{i}=a_{-i}$ ).

For a mode that is well resolved, the filtered mode shape function $\tilde{\mathbf{q}}(r)$ will be small compared to the original shape function $\mathbf{q}(r)$ itself. On the other hand, if an eigenvector is dominated by point-to-point oscillations then $\tilde{\mathbf{q}}(r)$ will be as large as $\mathbf{q}(r)$. Hence by comparing the magnitude of $\tilde{\mathbf{q}}(r)$ with $\mathbf{q}(r)$ we can define a quantitative measure of how well resolved a mode is. Here this is done by calculating

$$
\sigma=\|\tilde{\mathbf{q}}\| /\|\mathbf{q}\|, \quad \text { where }\|f\|^{2}=\frac{1}{2} \sum_{n=1}^{N-1}\left(r_{n+1}-r_{n}\right)\left[r_{n} f\left(r_{n}\right)^{2}+r_{n+1} f\left(r_{n+1}\right)^{2}\right] \text {, }
$$

which corresponds to the ratio between the $L^{2}$ norms of the filtered and original shape functions. In this definition the norm is calculated using the trapezoidal rule. The modes are ordered by increasing $\sigma$ and only a given number of well-resolved modes are used. The filter used in this work is the 7-point filter proposed in $[23]$.

\subsection{Identification of acoustic modes}

The eigenvalue problem (2) yields not only acoustic modes but also entropy and vorticity modes. With uniform base flow the distinction between these three types of modes is unambiguous. Vorticity modes have 
no influence on the perturbations of pressure or density, and are associated with divergence-free velocity fields. Entropy modes generate density fluctuations but no velocity or pressure fluctuation. With parallel sheared flow this separation is not possible, but the modes can still be categorised as predominantly associated with acoustic, vorticity or entropy fluctuations.

To identify pressure dominated modes we can calculate

$$
\chi=\left\|p^{\prime}\right\| /\left(\left\|p^{\prime}\right\|^{2}+\left\|\mathbf{u}^{\prime}\right\|^{2}+\left\|\rho^{\prime}\right\|^{2}\right)^{1 / 2} .
$$

If this quantity is above a certain threshold the mode is considered to be pressure dominated (so mostly acoustic) and is retained. Note that the definition above applies when the physical quantities are nondimensional.

Another method to separate acoustic modes from the convected modes is based on the axial wavenumber $k$. For acoustic modes $\operatorname{Re}(k)$ is expected to remain bounded above by $\omega / \min \left(u_{0}+c_{0}\right)$ while for convected modes we can expect $\omega / \max \left(u_{0}\right) \lesssim \operatorname{Re}(k) \lesssim \omega / \min \left(u_{0}\right)$ which corresponds to the continuous spectrum. In this work we discard modes for which $\operatorname{Re}(k)>k_{\max }$ where $k_{\max }$ is the midway point between $\omega / \min \left(u_{0}+c_{0}\right)$ and $\omega / \max \left(u_{0}\right)$.

\subsection{Direction of propagation}

In this work, the direction of propagation of a given mode is identified by the direction of decay, i.e. the sign of $\operatorname{Im}(k)$. This method does not address instability waves (such as those discussed by Rienstra, Brambley and Peake [27, 28]) but the focus in the present paper is on standard acoustic modes for which this simplified method is sufficient. A more general technique consists in estimating the group velocity of each mode by performing a second calculation with a small imaginary part added to the frequency. This would double the cost of the calculations but offer the advantage of taking into account the influence of the variations of the impedance function $Z(\omega)$ with frequency.

\subsection{Ordering of the acoustic modes}

Finally the acoustic modes are ordered based on the following definition of the radial wavenumber:

$$
k_{r}=\sqrt{\left(\omega-u_{0} k\right)^{2} / c_{0}^{2}}-k^{2}
$$

It should be noted that this definition is only valid for a uniform mean flow, since it is derived from the dispersion relation for acoustic waves in uniform flow. It is still useful to define this quantity when a boundary layer is included in the mean flow profile. For a given azimuthal order $m$ the modes are ordered based on the real part of $k_{r}$.

\subsection{Validation}

The numerical model and its Matlab implementation have been validated against various existing solutions, including analytical solutions for a rigid duct with uniform flow, numerical solutions for cases with uniform flow and the Myers condition [29] and numerical solutions from [17] obtained with the Myers condition, the modified impedance condition (6) and a fully-resolved boundary layer. In all cases very good agreement was observed with the reference solutions.

\section{Parametric Study}

To assess the effect of the boundary layer on liner performance in modern turbofan engines, the attenuation of duct modes in a straight circular duct is calculated over a wide range of parameters. All test cases are defined in terms of non-dimensional parameters by setting the mean density, sound speed and duct radius to 1 .

Except for the boundary layer $(R-\delta<r<R)$, the mean flow is uniform with axial velocity $u_{0}=M c_{0}$ where $M$ is the mean flow Mach number. Three flow conditions are typically considered corresponding to 


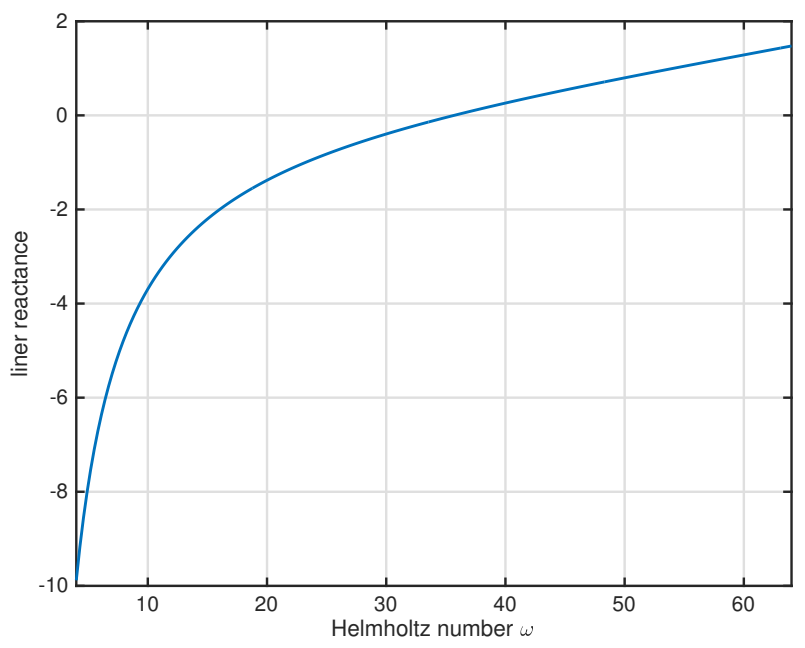

Figure 1: Non-dimensional liner reactance $X_{\mathrm{L}}(\omega)$ as a function of the Helmholtz number $\omega$.

the certification points. We will primarily use the cutback $(M=0.5)$ and sideline $(M=0.55)$ conditions where the flow velocity is high. The actual grazing flow velocity 'seen' by the intake liner can be much larger than the Mach number at the fan face, especially close to the throat of the inlet. So a wide range of Mach numbers will be considered in section 5.3.

The boundary layer thickness varies substantially over the length of the intake liner. In the bypass duct the boundary layer is also expected to be relatively thick. Most of the calculations will be with a linear velocity profile. While a linear profile is unlikely to be realistic the actual velocity profile of the boundary layer was found to have a limited impact on the liner attenuation [8, 18] provided that the displacement thickness $\delta_{1}$ defined in (7) remains constant (This observation will be tested again using different boundary layer profiles). We therefore consider a range of values for the displacement thickness of the boundary layer from $0.25 \%$ to $1.5 \%$. There is little information on the detailed profiles of the boundary layers found in the intake and bypass ducts, but the case $\delta_{1}=1 \%$ will be considered as a best estimate for the boundary layer found in front of the fan. Smaller values of $\delta_{1}$ should be expected upstream of the fan where the boundary layer is growing, and values above $1 \%$ should be representative of the boundary layer inside the bypass duct.

We use a simple impedance model for a single-degree-of-freedom liner [30]:

$$
\frac{Z}{\rho_{0} c_{0}}=R_{\mathrm{L}}+\mathrm{i} X_{\mathrm{L}}(\omega)
$$

The baseline values for the liner resistance are $R_{\mathrm{L}}=4$ and 5 for the cutback and sideline configurations, respectively, but other values of $R_{\mathrm{L}}$ will also be considered to illustrate the effect of the liner resistance. The reactance $X_{\mathrm{L}}$ is shown in figure 1 and the specific values for the different configurations will be given below. These values of impedance have been chosen to be representative of a typical intake liner.

\section{Individual modes}

First we focus on the attenuation rate of duct modes taken individually. For this purpose results are presented for the Blade Passing Frequency (BPF). These results are therefore relevant for tonal noise, typically fan noise. For the cutback and sideline configurations the BPF corresponds to a Helmholtz number (based on the duct radius) of 23 and 28.

\subsection{Intake (upstream propagation)}

We begin by discussing the case of the intake liner, by considering only the modes propagating against the flow. Rather than considering a single duct mode, we assess the effect of the boundary layer over a 


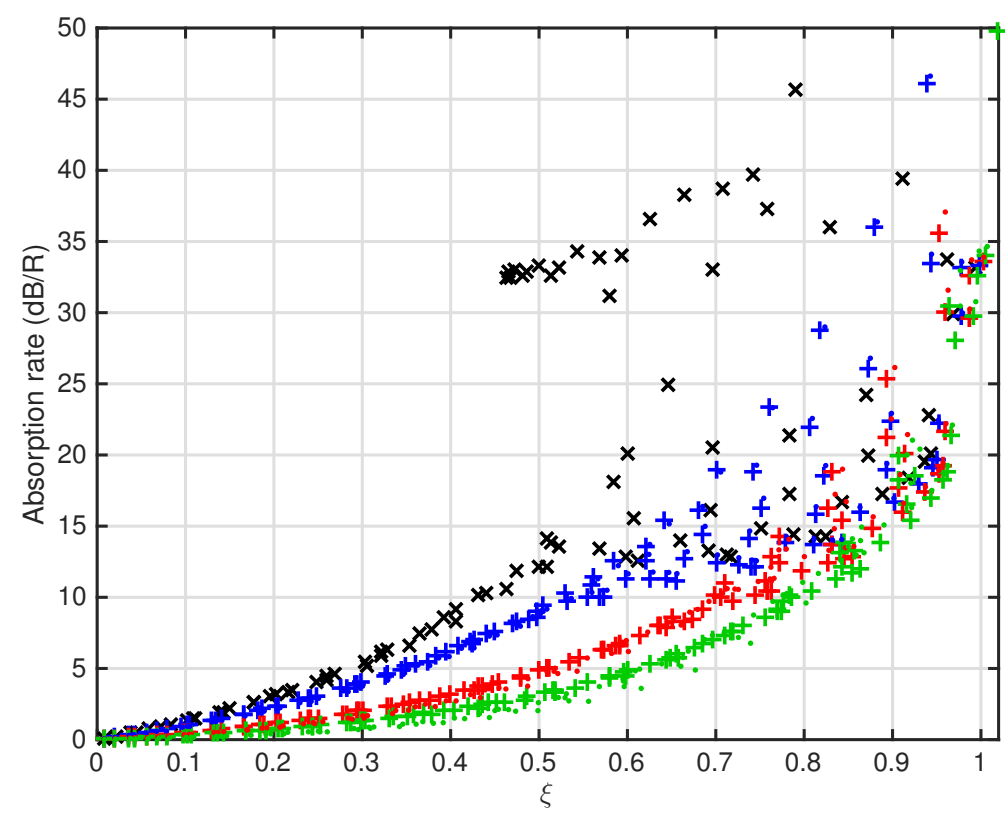

Figure 2: Absorption rate as a function of $\xi$ for the modes propagating upstream with $M=0.5, \omega=23$ and the liner model (15) with $R_{\mathrm{L}}=4$ corresponding to $Z \simeq 4-\mathrm{i}\left(m_{\max }=28\right)$. Boundary layer thickness $\delta_{1}=0$ (black), $0.5 \%$ (blue), $1 \%$ (red), $1.5 \%$ (green). Fully-resolved boundary layer $(\cdot)$, Myers impedance condition $(\times)$ and modified impedance condition $(6)(+)$.

range of modes, by solving the eigenvalue problem (12) for a large number of radial modes and for all the azimuthal orders up to $m=m_{\max }$.

When studying the attenuation of acoustic modes in lined ducts, Rice [13] introduced the following cut-off ratio:

$$
\eta=\frac{\omega / c_{0}}{\left|k_{r}\right|}\left[\left(1-M^{2}\right) \cos (2 \phi)\right]^{-1 / 2}
$$

where $\phi$ is the phase of $k_{r}$. For hard-wall ducts $(\phi=0), \eta>1$ for propagating modes and $\eta<1$ for evanescent modes. For lined ducts, $k_{r}$ is complex valued $(\phi \neq 0)$ and there is a continuous transition from well cut-on modes to strongly cut-off modes but $\eta$ remains a useful parameter correlated to the attenuation rate of the mode.

In the present work we use a similar parameter defined as follows

$$
\xi=\eta^{-2}=\operatorname{Re}\left[\left(1-M^{2}\right) \frac{k_{r}^{2}}{\omega^{2} / c_{0}^{2}}\right] .
$$

The reasons for introducing this parameter are that (i) we found modes corresponding to complex values of $\eta$ while $\xi$ remains real and (ii) almost all the modes of interest are found in the range $0<\xi<1$.

To identify general trends in the absorption rate and in the influence of the boundary layer Figure 2 shows the absorption rate of the duct modes as a function of the parameter $\xi$ for the cutback configuration $(M=0.5)$ at the blade passing frequency and with the liner model (15), corresponding to an impedance $Z \simeq 4-$ i. Results are shown for the Myers condition (4), the modified impedance condition (6) and the fully-resolved boundary layer. Each data point in Figure 2 corresponds to an individual mode calculated with a particular boundary condition and for a given boundary layer thickness.

A first observation is that when the attenuation is smaller than approximately $12 \mathrm{~dB} / \mathrm{R}$ there is a well defined trend relating the mode absorption and $\xi$. Above this threshold the modal absorption behaves more erratically. Close to cut-off $(\xi=1)$, for large absorption, or for a thick boundary layer, this trend is not so clearly visible, which could be related to the fact that these modes tend to be very sensitive to any change 

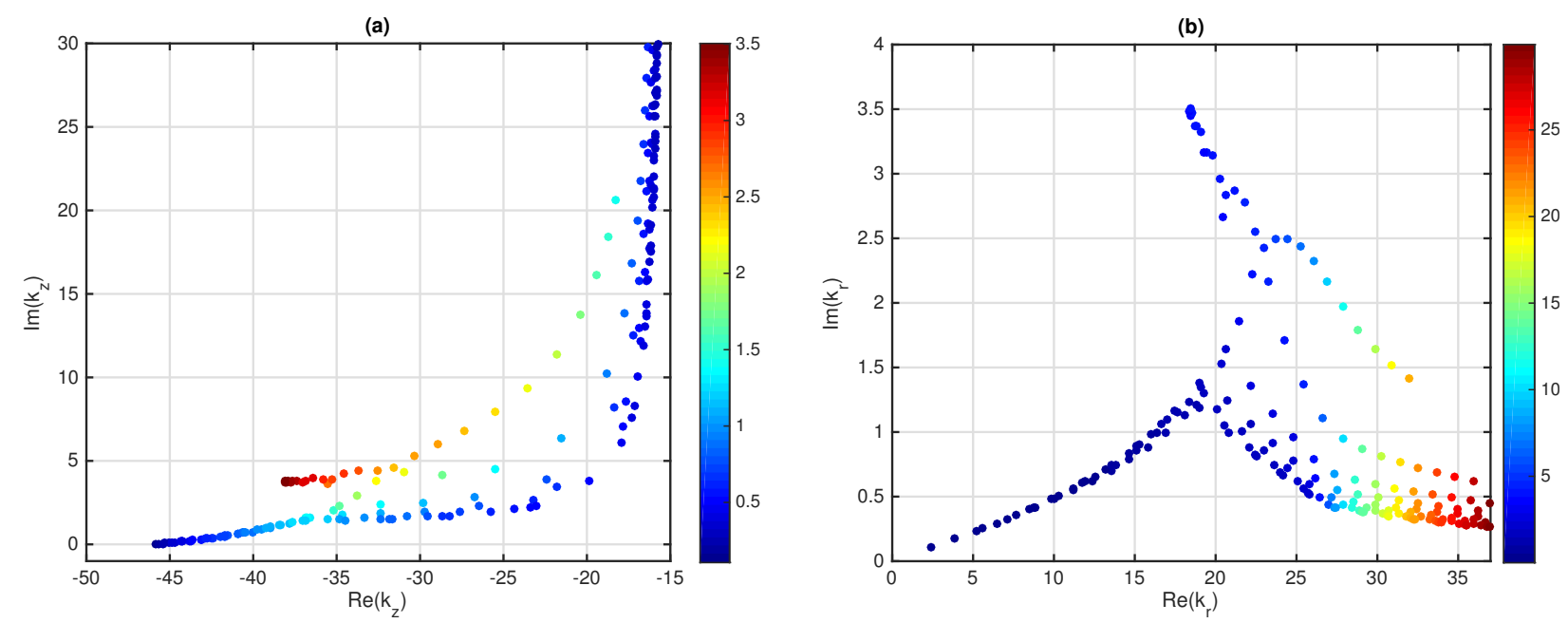

Figure 3: Modes propagating upstream calculated using the Myers condition for $M=0.5, \omega=23$ and the liner model (15) with $R_{\mathrm{L}}=4$. (a) Axial wavenumbers $k_{z}$ with colors representing $\operatorname{Im}\left(k_{r}\right)$. (b) Radial wavenumbers $k_{r}$ with colors representing $\operatorname{Im}\left(k_{z}\right)$.

in parameters. Obviously one should expect the absorption to be close to zero for the plane wave $(\xi=0)$ and to increase with $\xi$, but figure 2 indicates that this trend follows a precise relation, where the absorption increases with $\xi$, at first relatively slowly, but then quite sharply for modes close to cut-off.

With the Myers condition, there is a cluster of modes with an absorption rate between 30 and $35 \mathrm{~dB}$ and a $\xi$ close to 0.5 . The nature of these modes is further described by plotting the axial and radial wavenumbers in the complex plane, as shown in Figure 3. For these modes the imaginary part of the radial wavenumber is relatively large but still comparable to the other modes. For surface waves $\operatorname{Im}\left(k_{r}\right)$ is expected to be much larger. So, although they begin to show properties that are expected of surface waves, the group of modes observed in figure 2 can still be considered as acoustic modes. It is important to note that surface waves do not represent a category of modes completely distinct from classical acoustic modes: when vayring the impedance an acoustic mode can smoothly evolve into a surface wave.

There is a clear trend for the impact of the boundary layer on the absorption with a consistent reduction of the attenuation as the boundary layer thickness increases, especially between $\delta_{1}=0.5 \%$ and $1 \%$. The change in attenuation is significant, for many modes the reduction can be of the order of 5 or $10 \mathrm{~dB}$. This trend is properly captured by the modified impedance condition (6), including for the thick boundary layer $\left(\delta_{1}=1.5 \%\right)$. Even when the absorption rate is above $12 \mathrm{~dB}$, this impedance condition is able to provide predictions that are relatively close to the exact solution.

This reduction in attenuation associated with thicker boundary layers is expected for intake liners. It is generally explained by the refraction effect of the mean flow shear in the boundary layer. For modes propagating upstream, sound is refracted away from the duct wall, thus resulting in a reduction of the effective attenuation achieved by the liner.

However this overall trend in the effect of the boundary layer can change by simply considering a different liner. In figure 4 we perform a similar analysis but with a lower liner resistance $R_{\mathrm{L}}=2$.

In this case the difference between the Myers condition and the model with a finite-thickness boundary layer is also significant. For instance for modes with $\xi \simeq 0.6$ the Myers condition underestimates the attenuation by more than $5 \mathrm{~dB}$ compared to a boundary layer thickness $\delta_{1}=1 \%$. The modified impedance condition (6) is able to capture more accurately the absorption and the effect of the boundary layer. This is particularly true for the boundary layer thickness $0.5 \%$, and less so for the larger values of $\delta_{1}$.

The key difference when compared to the impedance $Z \simeq 4-\mathrm{i}$ used in figure 2 is that with $Z \simeq 2-\mathrm{i}$ we do not observe a consistent reduction of the attenuation for increasing boundary layer thickness. Instead the attenuation increases between $\delta_{1}=0,0.5$ and $1 \%$ but then decreases when the boundary layer thickness 


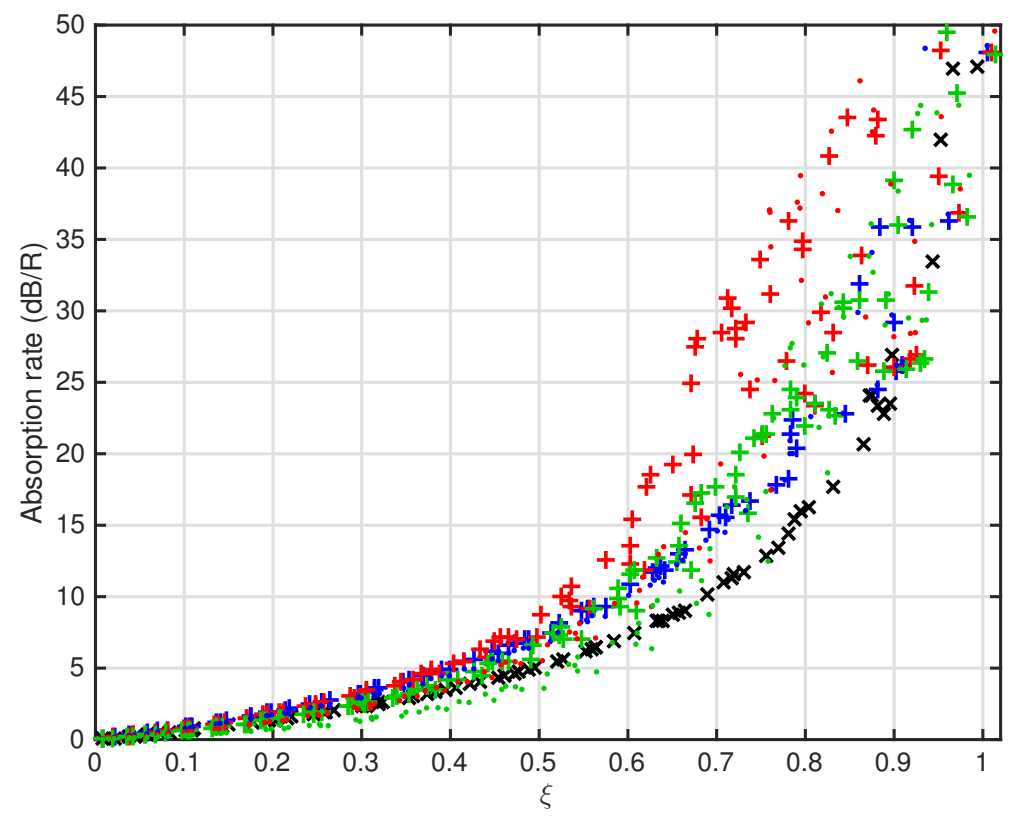

Figure 4: Absorption rate as a function of $\xi$ for the modes propagating upstream with $M=0.5, \omega=23$ and an impedance $Z=2-\mathrm{i}\left(m_{\max }=28\right)$. Boundary layer thickness $\delta_{1}=0$ (black), $0.5 \%$ (blue), $1 \%$ (red), $1.5 \%$ (green). Fully-resolved boundary layer $(\cdot)$, Myers impedance condition $(\times)$ and modified impedance condition $(6)(+)$.

is further increased to $1.5 \%$. This behaviour cannot be explained by the refraction effect of the boundary layer, and this illustrates that the effect of boundary layer on the liner attenuation is more complex than only changing the propagation of sound before it interacts with the liner. The fact that the trends in figures 2 and 4 are reversed suggests that there is an impedance for which the boundary layer has no, or little, effect on attenuation.

Note also that with $R_{\mathrm{L}}=2$ the link between absorption and cut-off ratio already observed in figure 2 is even more clearly visible. This is especially true for the Myers condition for which all the modes lie on a single curve.

It is also possible to consider modes individually to better understand their properties. As an example results are shown for the sideline configuration $(\omega=28$ and $M=0.55)$ at the blade passing frequency with a boundary layer thickness $\delta_{1}=1 \%$ and the liner model (15) with $R_{\mathrm{L}}=5$ corresponding to $Z \simeq 5-0.56 \mathrm{i}$. Figure 5 shows the absorption rate for each mode ordered by azimuthal and radial orders. Also shown is the difference between the different predictions.

It can be seen that the attenuation increases with both the azimuthal and radial orders. This is expected and is consistent with the results presented above. The Myers condition (4) tends to significantly overestimate the attenuation. The difference with the fully resolved boundary layer increases with azimuthal order to reach a maximum (when $\xi$ is close to 1) and then decay. For instance for the first radial mode with $m=24$ the error is more than $30 \mathrm{~dB}$, and the error is above $5 \mathrm{~dB}$ for a wide range of azimuthal orders. Again the modified impedance condition (8) provides significantly improved predictions, with differences with the reference solutions of at most $4 \mathrm{~dB}$.

We can assess more precisely the effect of the boundary layer on a specific mode, for instance the first radial mode $m=24$ which is representative of the modes generated by the fan. Figure 6 shows how the attenuation rate of this mode varies with the boundary layer thickness, for the sideline case at the blade passing frequency.

With the baseline liner resistance $R_{\mathrm{L}}=5$ we can see a rapid decrease in attenuation when $\delta_{1}$ is increased with more than $30 \mathrm{~dB}$ between 0 and $1.5 \%$. Again this trends is consistent with the refraction effect of the boundary layer on waves propagating upstream. The Myers condition doesn't capture this trend and only 

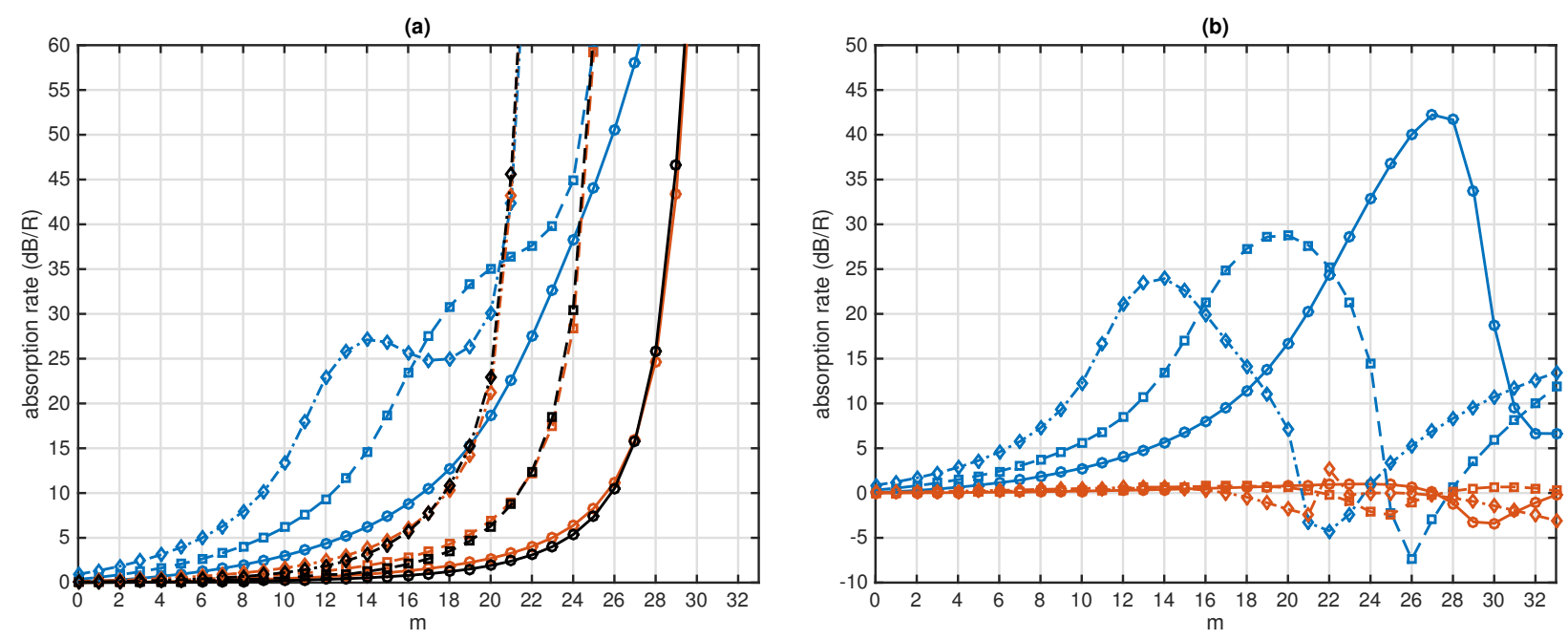

Figure 5: Modal attenuation as a function of the azimuthal order and radial order for the sideline case $(\omega=28$ and $M=0.55)$ with $\delta_{1}=1 \%$ and the liner model (15) corresponding to $Z \simeq 5-0.56 \mathrm{i}$. First (o), second $(\square)$ and third $(\diamond)$ radial modes. (a) Absorption rate in $\mathrm{dB} / \mathrm{R}$ predicted with the Myers condition (4) (blue), modified impedance condition (6) (red) and the fully-resolved boundary layer (black). (b) Difference between the predicted and exact absorption rate in dB/R.

predicts an attenuation independent of $\delta_{1}$. The modified impedance condition is able to follow quite closely the exact result.

With a lower liner resistance $R_{\mathrm{L}}=2$ the effect of the boundary layer thickness is noticeably different. From $\delta=0 \%$ to $0.4 \%$ the boundary layer increases the attenuation of this mode, which goes against an interpretation in terms of refraction effect. For larger values of $\delta_{1}$ the trend is a reduction of the attenuation. The modified impedance condition is able to represent the overall trend but overestimates the attenuation by 3 or $4 \mathrm{~dB}$.

Finally it is also interesting to consider the change in mode shape functions associated with the presence of the boundary layer. This is shown in figure 7 for the first radial mode with $m=24$ for the sideline conditions at the blade passing frequency. Since these shape functions are defined up to a complex-valued factor, care is needed to perform a meaningful comparison. All the modes are normalised so that the integrated $L^{2}$ norm of the pressure shape function is unity. The phase of the normalisation constant for each mode is then determined so as to minimise the difference with the pressure shape function obtained for the fully-resolved boundary layer with $\delta_{1}=1 \%$. It can be seen in figure 7 that the boundary layer changes the shape of the mode with the two impedance values considered. Whether this change is significant is an open question and will probably depend on the application (mode matching schemes, mode detection techniques, etc). The general evolution of the mode shape with $\delta_{1}$ is captured by the modified impedance condition.

\subsection{Bypass duct (downstream propagation)}

The overall picture changes significantly for modes propagating downstream. Figure 8 shows the attenuation rate against $\xi$ for the cutback configuration at 1 BPF. In contrast with the upstream modes, which tend to follow a single well-defined relation between absorption and $\xi$, two different behaviours are observed for the downstream modes. For most of the modes the attenuation is relatively small, and more importantly, the boundary layer has very little impact on the attenuation.

The second group of modes has a much stronger attenuation and the boundary layer has a noticeable effect (in the examples in figure 8 the attenuation increases with $\delta_{1}$ ). For instance with $R_{\mathrm{L}}=1$ for $\xi \simeq 0.5$ there is a $10 \mathrm{~dB}$ difference between the Myers condition and the solution with a $1 \%$ boundary layer thickness. The corresponding axial and radial wavenumbers are plotted in the complex plane in figure 9. This second group of modes has radial wavenumbers with large imaginary parts and appear as a distinct set of modes in the $k_{z}$ complex plane. The corresponding mode shape functions decay rapidly away from the liner, and these modes are therefore behaving like surface waves. 

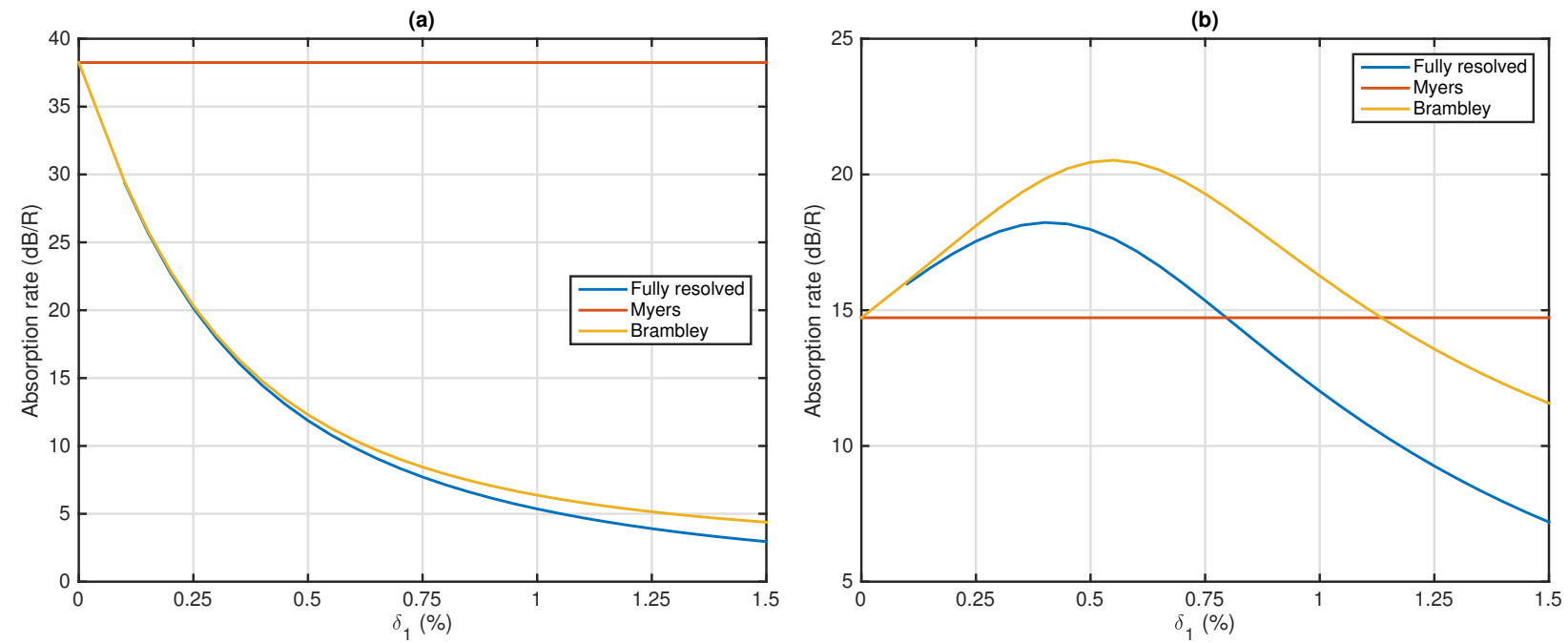

Figure 6: Absorption rate in $\mathrm{dB} / \mathrm{R}$ as a function of the boundary layer thickness for the first radial mode with $m=24$ for the sideline $\left(\omega=28\right.$ and $M=0.55$ ) case with the liner model (15) with (a) $R_{\mathrm{L}}=5$ and (b) $R_{\mathrm{L}}=2$.

(a)

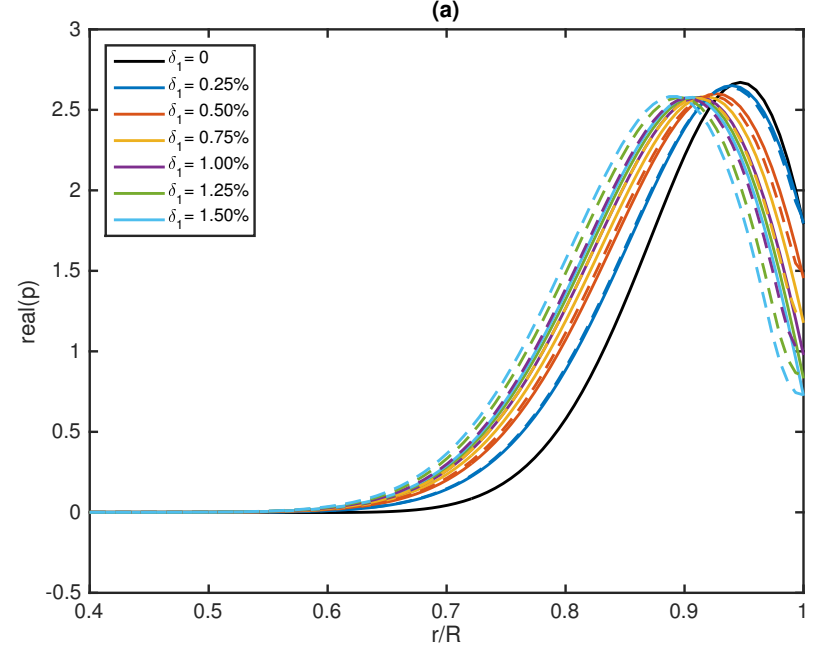

(b)

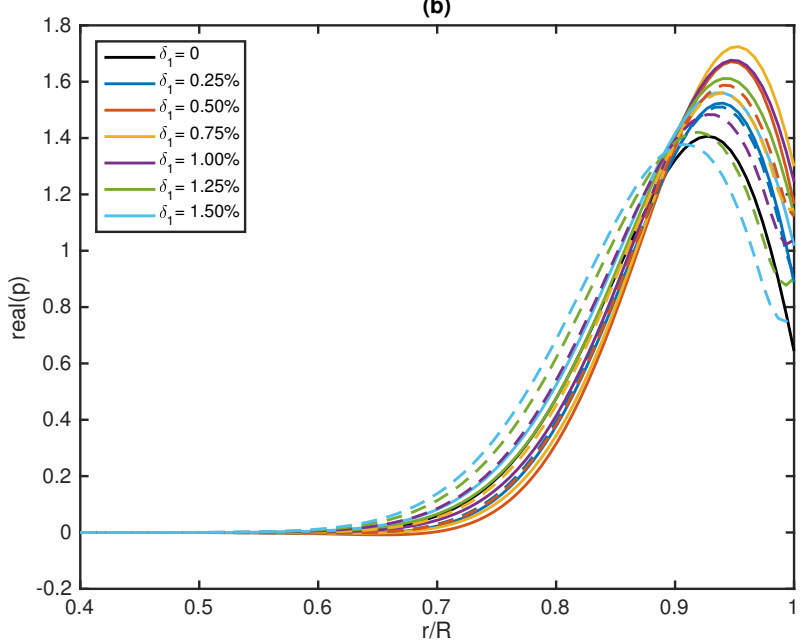

Figure 7: Mode shape function of the first radial mode with $m=24$ for the sideline case with the liner model (15) with (a) $R_{\mathrm{L}}=5$ and (b) $R_{\mathrm{L}}=2$. Myers condition: solid black line. Fully-resolved boundary layer: solid color lines. Modified impedance condition: dashed lines. 

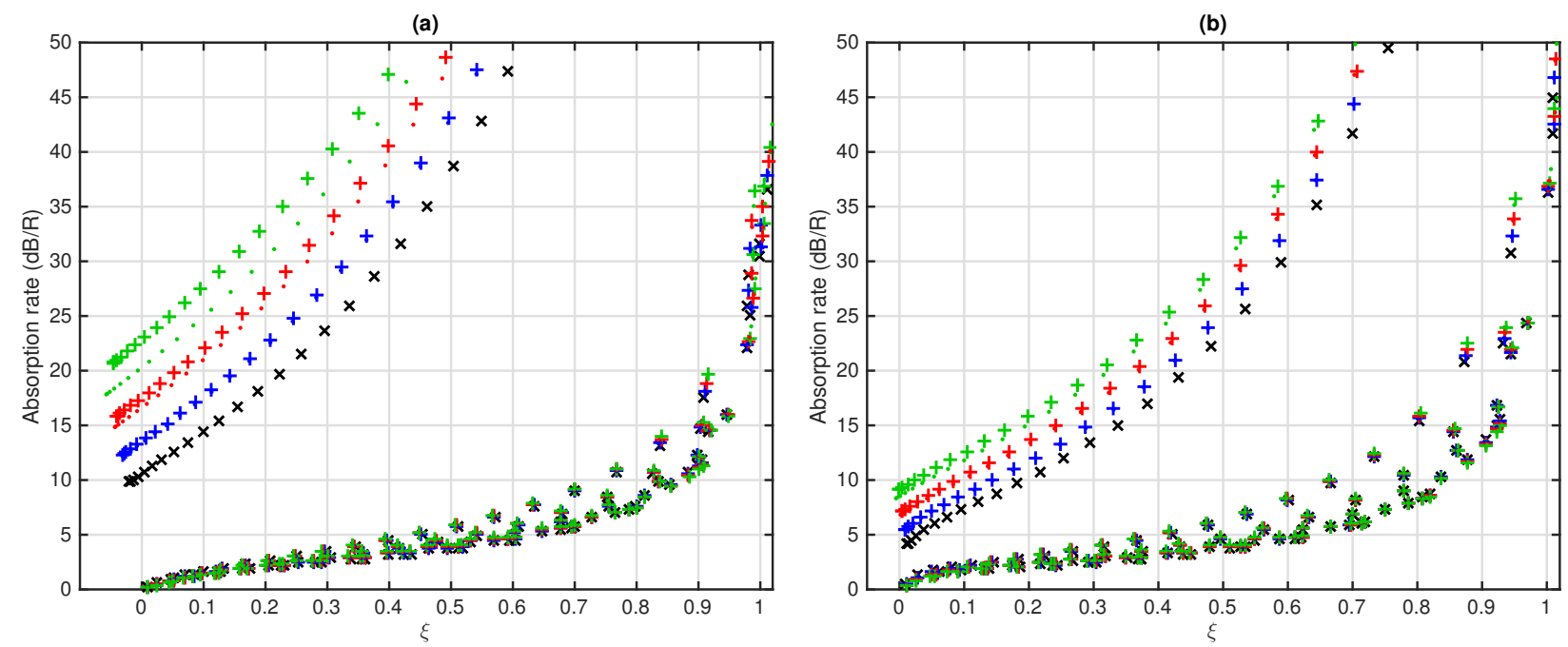

Figure 8: Absorption rate as a function of $\xi$ for the modes propagating downstream with $M=0.5, \omega=23$ with the liner model (15) with (a) $R_{\mathrm{L}}=1$ or (b) $R_{\mathrm{L}}=2$. Boundary layer thickness $\delta_{1}=0$ (black), $0.5 \%$ (blue), $1 \%$ (red), $1.5 \%$ (green). Fully-resolved boundary layer $(\cdot)$, Myers impedance condition $(\times)$ and modified impedance condition $(6)(+)$.
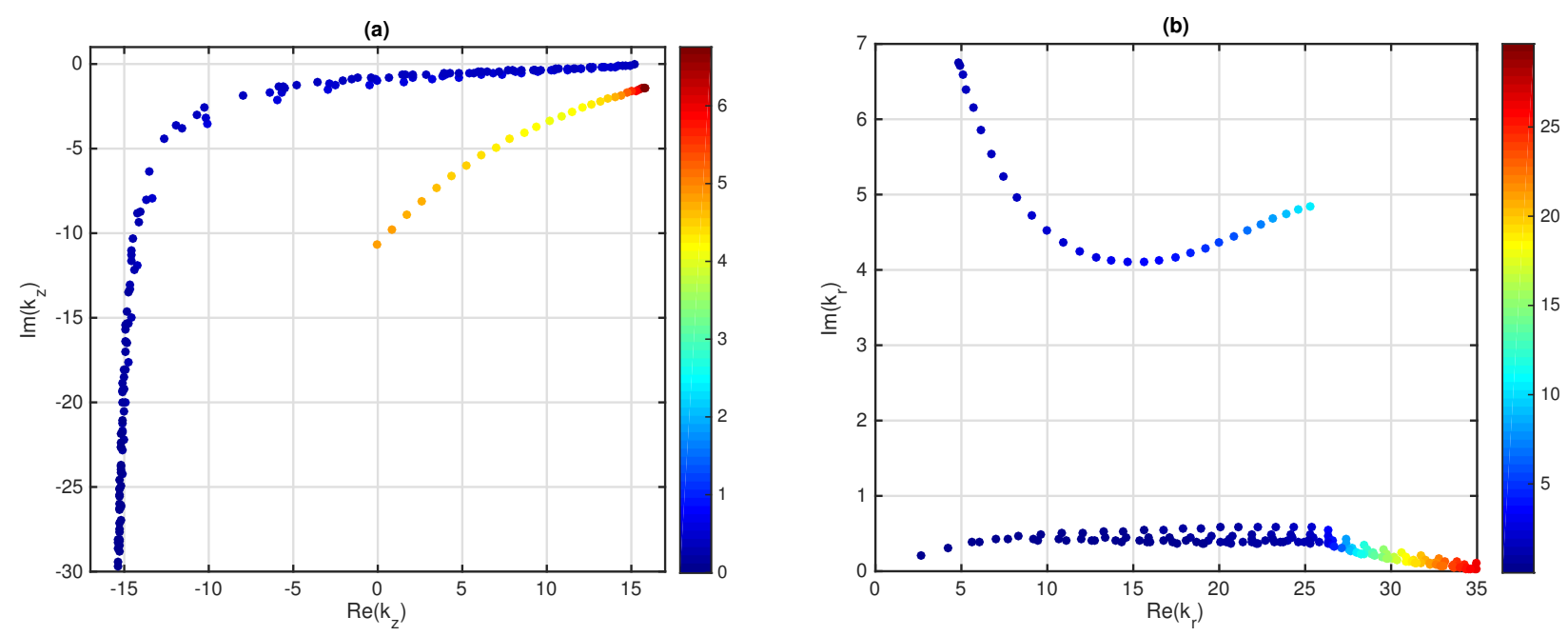

Figure 9: Modes propagating upstream calculated using a fully resolved boundary layer for $\delta_{1}=0.5 \%, M=0.5, \omega=23$ and the liner model (15) with $R_{\mathrm{L}}=1$. (a) Axial wavenumbers $k_{z}$ with colors representing $\operatorname{Im}\left(k_{r}\right)$. (b) Radial wavenumbers $k_{r}$ with colors representing $-\operatorname{Im}\left(k_{z}\right)$. 
(a)

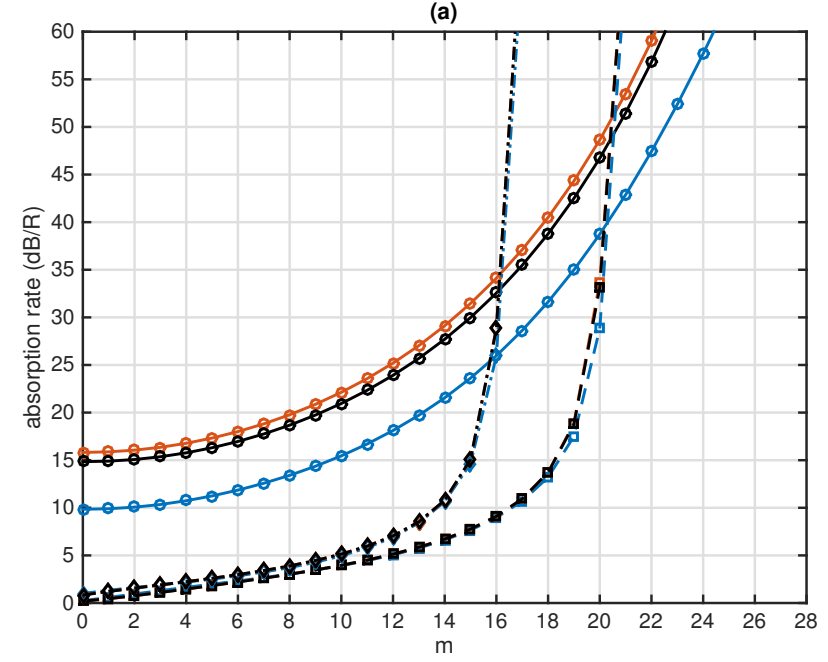

(b)

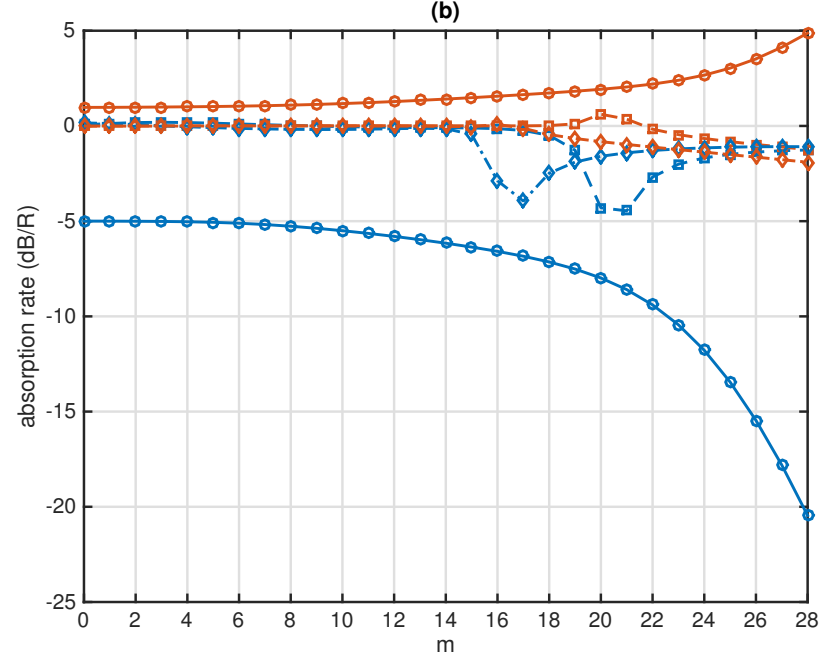

Figure 10: (a) Absorption rate in $\mathrm{dB} / \mathrm{R}$ as a function of the azimuthal order. (b) Difference between the impedance conditions and the fully-resolved boundary layer. Blue: Myers condition (4). Red: modified impedance condition (6). Black: fully-resolved boundary layer. First (०), second $(\square)$ and third $(\diamond)$ radial modes. Cutback configuration $(\omega=23$ and $M=0.5)$ with $\delta_{1}=1 \%$ and the liner model (15) with $R_{\mathrm{L}}=1$.

Figure 10 presents the modal attenuation for the different azimuthal and radial orders with the liner resistance $R_{\mathrm{L}}=1$ and a boundary layer thickness $\delta_{1}=1 \%$. Based on the mode ordering defined in section 3 the surface waves are found as the first radial mode of each azimuthal order. As seen in figure 8, the attenuation of these modes is significantly higher than the other modes, except for the azimuthal orders close to cut-off. The Myers condition consistently underestimates the attenuation of the surface waves wheras the modified impedance condition (6) is found to be in good agreement with the fully-resolved boundary layer. In particular the effect of the boundary layer on the surface waves is accurately captured.

We consider again the first radial mode with $m=24$ and in figure 11 we plot its attenuation as a function of the boundary layer thickness for $R_{\mathrm{L}}=1$ and 2. The consistent underprediction of the Myers condition is clearly visible. As expected in the case of downstream propagation, with a thicker boundary layer the attenuation of the mode is increased which is consistent with the refraction effect of the mean flow shear turning the waves towards the liner. The same trends are observed for other azimuthal orders $m$.

\subsection{Influence of the flow velocity}

To assess the effect of the mean flow on the attenuation, the cutback configuration $\left(\omega=23\right.$ with $R_{\mathrm{L}}=4$ and $\delta_{1}=1 \%$ ) is used and the mean flow velocity is varied from $M=0$ (no flow) to $M=0.7$. Results are shown in figure 12 for the upstream modes (results for the downstream modes do not bring more insight).

Let us first consider the results with the fully-resolved boundary layer. We see that for low Mach numbers (and with no flow) the upstream modes present a similar trend as the downstream modes discussed in section 5.2. As observed in figure 8, a distinct group of modes exhibits a stronger rate of attenuation. These are modes with low radial orders, similar to surface waves, and for which the rate of attenuation increasing more rapidly with cut-off ratio. The other modes are not strongly influenced by the boundary layer. As we increase the Mach number we see these modes progressively collapsing on a single curved and we recover the trend observed in section 5.1. It is therefore important to note that for low Mach numbers the qualitative behaviour of the downstream and upstream modes is similar. It is only for higher Mach numbers (in this case above 0.4) that there is a clear difference in the behaviour of the upstream modes (i.e. all the modes are strongly influenced by the boundary layer).

Secondly, with the Myers condition we see that the group of modes with a stronger attenuation does not merge with the other modes as the flow velocity increases. Instead they move to increasingly higher attenuation, and correspond to the group of modes already observed in figure 2 . 
(a)

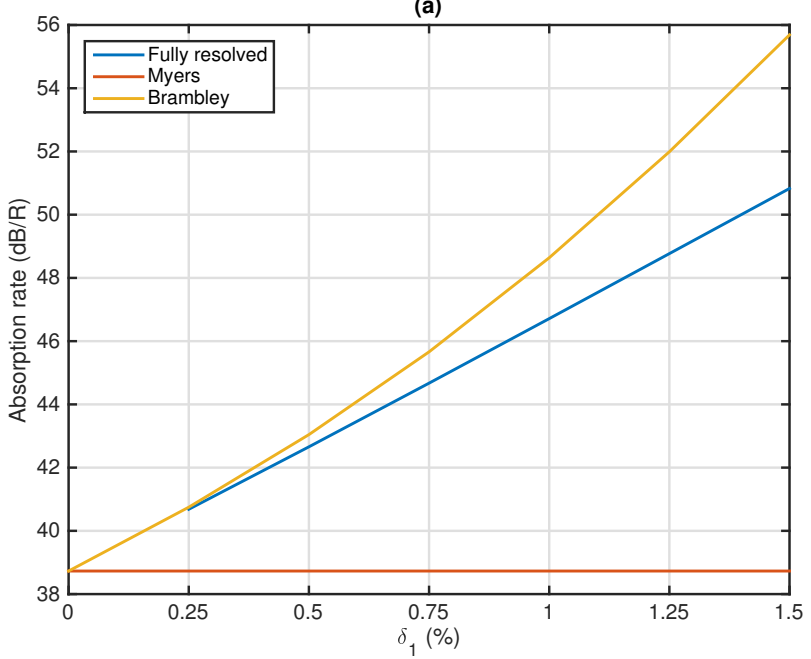

(b)

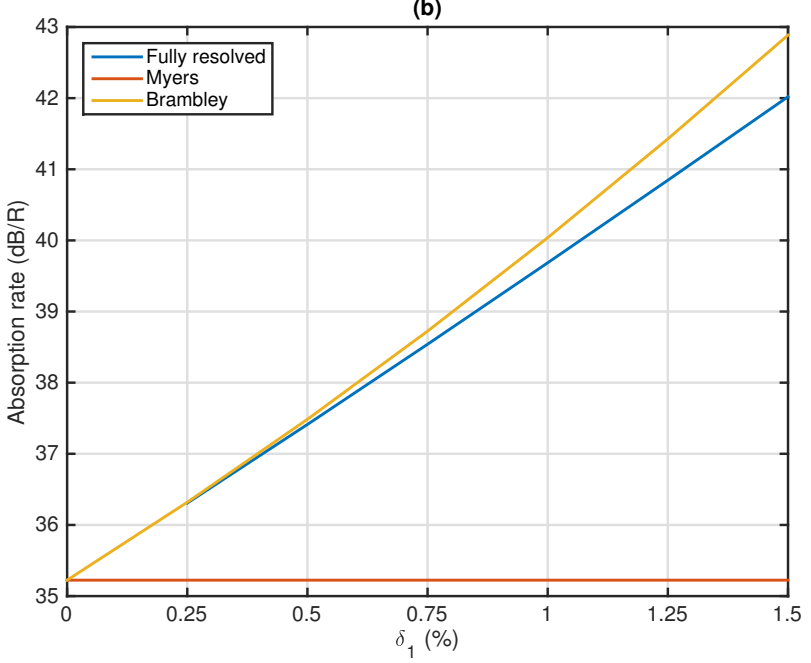

Figure 11: Absorption rate in $\mathrm{dB} / \mathrm{R}$ as a function of the boundary layer thickness for the first radial mode with $m=24$ for the cutback case $(\omega=23$ and $M=0.5)$ with the liner model (15) with (a) $R_{\mathrm{L}}=1$ and (b) $R_{\mathrm{L}}=2$.

Thirdly, the modified impedance condition (6) follows the results of the fully-resolved boundary layer quite well, except for the highest Mach number where the attenuation is overestimated.

\section{Multi-mode broadband noise}

A simple model commonly used as an approximate description of broadband noise in intake and bypass ducts is that of uncorrelated modes with equal energy per mode. For a uniform flow it is relatively easy to show that the rate of attenuation of the acoustic power in the duct is given by the average of $\mathrm{e}^{ \pm 2 \operatorname{Im}\left(k_{m}\right)}$ calculated over all the modes carrying energy. The \pm sign changes when downstream or upstream modes are considered. Since lengths are normalised by the duct radius, this represents an attenuation rate in $\mathrm{dB}$ per radius. For hard-walled ducts there is a finite number of propagating modes, and evanescent modes are excluded, so the number of modes to include is well defined. With a lined walls this is not the case, and we have to define which modes should be included. Here we exclude the modes for which $\xi$ is larger than 1.2. Including more modes by increasing this threshold does not alter the trends in the results, but only shifts the attenuation rate up by allocating more energy to modes that are decaying very rapidly. It should also be noted that we will only consider the differences between different predictions of the attenuation rate, since the absolute levels are functions of the threshold applied for the cut-off ratio.

Results are presented in figure 13 for the cutback configuration $(M=0.5)$ with the liner model (15) for two different liner resistance $R_{\mathrm{L}}=4$ and 1 (see figure 13).

For modes propagating downstream, we can see that the rate of attenuation is larger for the low resistance liner $\left(R_{\mathrm{L}}=1\right)$, as expected. The impact of the boundary layer thickness on the liner performance is very small, and in fact almost invisible for $R_{\mathrm{L}}=1$. This is consistent with the observation made on individual modes in section 5.2 since most of the modes are only very slightly influenced by the boundary layer. For $R_{\mathrm{L}}=4$, the general trend is a small increase of the absorption with increasing $\delta_{1}$. Both the Myers condition (4) and the modified impedance condition (6) are able to capture these small variations quite well.

There is a more pronounced effect for modes propagating upstream. For the liner with a high resistance $R_{\mathrm{L}}=4$ (figure 13b) there is a clear trend where increasing the boundary layer thickness reduces the attenuation. Again this is typically explained by the refraction effect through the boundary layer that tends to shield the liner from the sound field. An exception to this is for a relatively thin boundary layer $\left(\delta_{1}=0.5 \%\right)$ between $\omega=9$ and 17 where we observe an increase in attenuation compared to the case with an infinitely thin boundary layer. The attenuation achieved by the liner decays rapidly with frequency above 

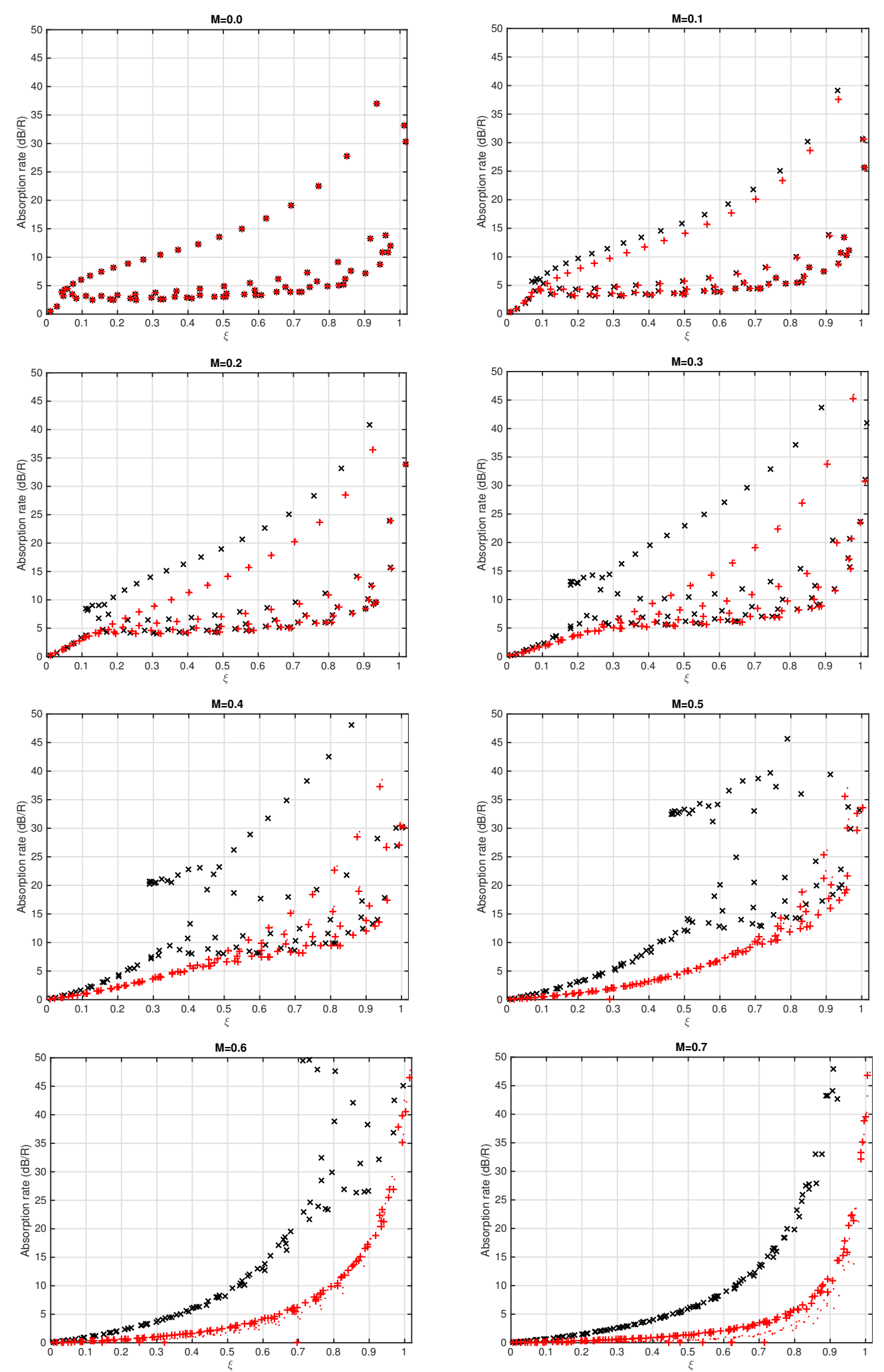

Figure 12: Attenuation of the upstream modes for the cutback condition $(\omega=23)$ with $R_{\mathrm{L}}=4$ and $\delta_{1}=1 \%$. Same notation as in figure 8 . 

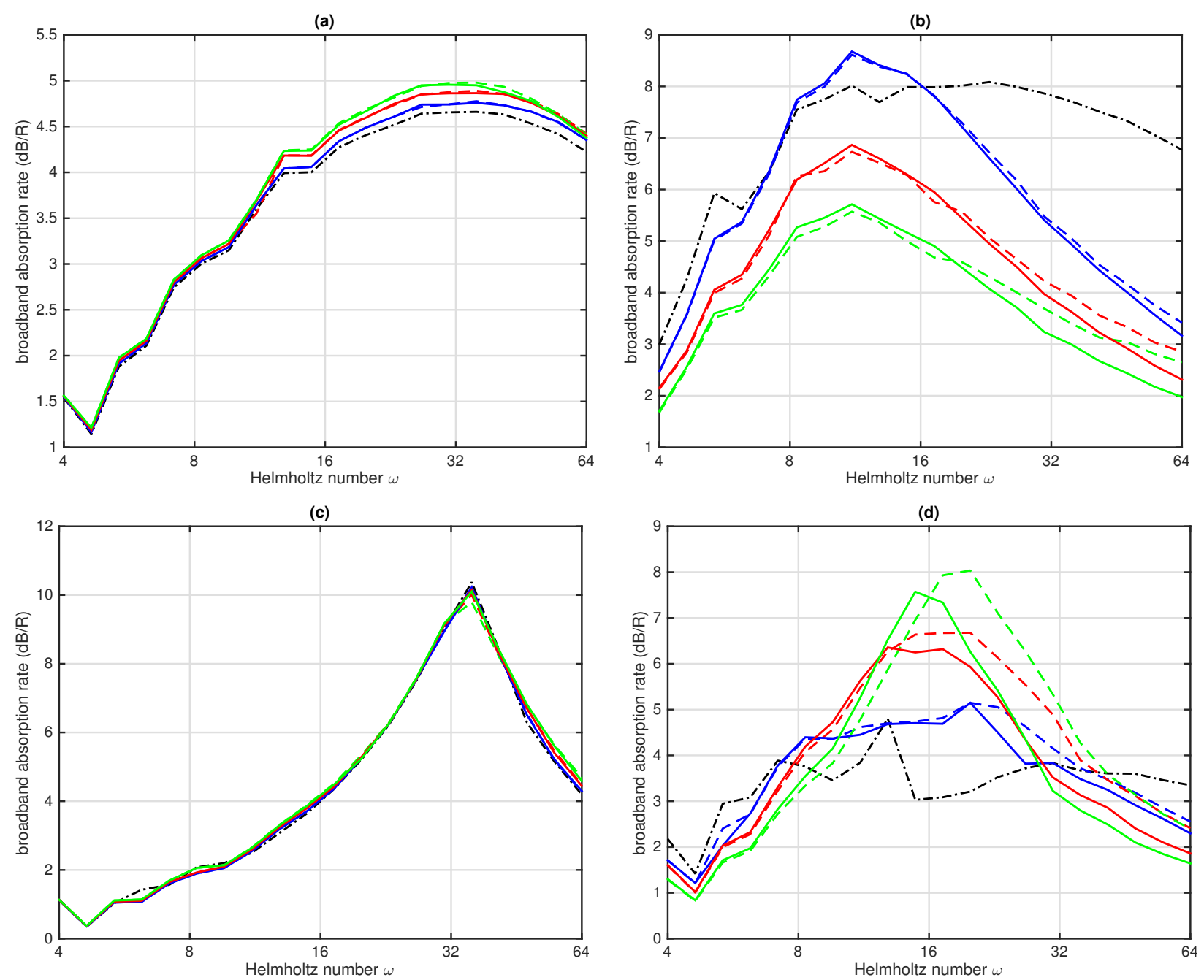

Figure 13: Broadband attenuation rate as a function of frequency $\omega$ for the modes propagating downstream (a,c) and upstream (b,d). Cutback condition $(M=0.5)$ with the liner model (15) with $R_{\mathrm{L}}=4$ (a,b) and $R_{\mathrm{L}}=1$ (c,d). Dot-dashed line: Myers condition (4). Solid line: Fully resolved boundary layer. Dashed line: Modified impedance condition (6). Boundary layer thickness $\delta_{1}=0.5 \%$ (blue), $1 \%$ (red) and $1.5 \%$ (green).

$\omega>10$, which is consistent with the refraction effect becoming stronger as the acoustic wavelength decreases compared to the boundary layer thickness. The modified impedance condition (6) is able to describe quite accurately the effect of the boundary layer over the frequency range considered in figure $13 \mathrm{~b}$ and for the three values of $\delta_{1}$ (the error in prediction remains under $1 \mathrm{~dB}$ ).

For the liner with a low resistance, in figure $13 \mathrm{~d}$, the change in attenuation with $\delta_{1}$ is more complex. For $\omega<10$ or $\omega>33$ we see a similar trend as before with a reduction in attenuation for thicker boundary layers. In between, the attenuation predicted with the Myers condition drops significantly while the attenuation calculated with a fully resolved boundary layer reaches a peak which becomes higher when $\delta_{1}$ is increased. As in figure $13 \mathrm{~b}$, there is a rapid reduction in attenuation with frequency above $\omega>17$. The modified impedance condition is able to capture the general trends seen with the fully-resolved boundary layer but does not capture the peak of attenuation as well as in figure $13 \mathrm{~b}$ (the difference still remains below $2 \mathrm{~dB}$ ). 


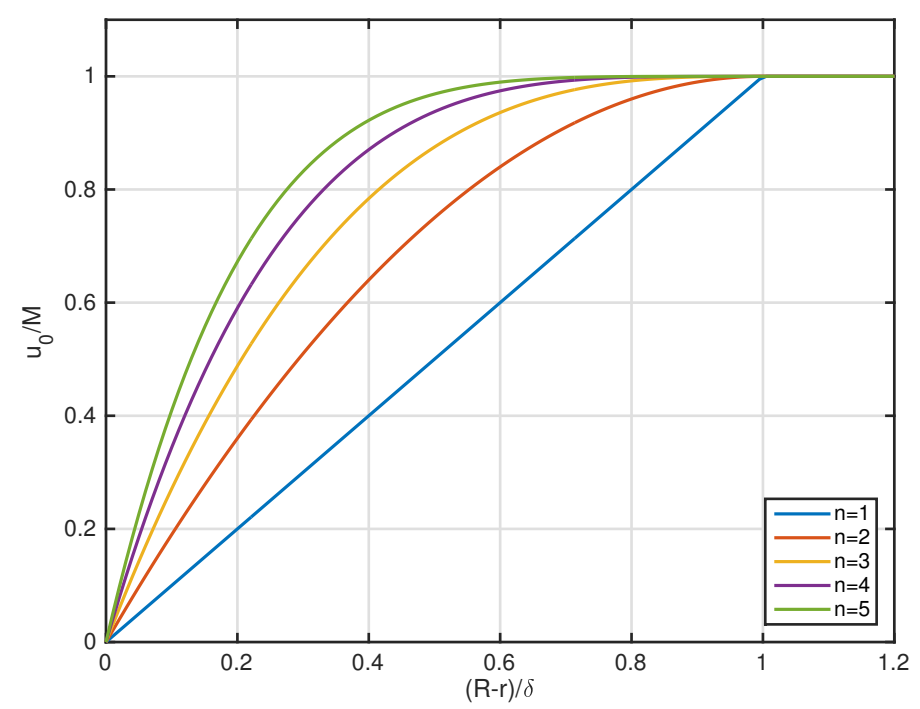

Figure 14: Boundary layer profiles (18) for a range of orders $n$.

\section{Influence of the boundary layer profile}

For individual modes it was observed in $[8,18]$ that the velocity profile of the boundary layer has little influence on the attenuation, provided that the displacement thickness $\delta_{1}$ remains constant. This is revisited here for a much larger number of modes. The velocity profile used for this purpose is

$$
\frac{u_{0}(r)}{M}=1-\left(1-\frac{R-r}{\delta}\right)^{n}, \quad \text { for } R-\delta \leqslant r \leqslant R,
$$

where $n$ can be adjusted to change the boundary layer profile. The case $n=1$ corresponds to the linear profile used so far. The displacement thickness $\delta_{1}$ defined in equation $(7)$ is $\delta_{1}=\delta /(1+n)$.

Figures 15 show the modal attenuation for the cutback configuration $(\omega=23$ and $M=0.5)$ with the liner model (15) with $R_{\mathrm{L}}=4$. In figures $15 \mathrm{a}$, b the total thickness $\delta$ is kept constant at $2 \%$, and we consider different boundary layer profiles with $n$ varying from 1 to 5 . As a consequence the displacement thickness varies from $\delta_{1}=1 \%$ to $0.333 \%$, with the boundary layer becoming thinner as $n$ increases. Results in figure 15 a show only a small change in attenuation for modes propagating downstream. The attenuation of the first radial modes is only slightly reduced when $n$ increases, and there is virtually no change for the other modes. However, for modes propagating against the flow, increasing $n$ leads to a significant increase in attenuation (for instance almost $10 \mathrm{~dB}$ for $\xi \simeq 0.6$ ).

In figures $15 \mathrm{c}, \mathrm{d}$, similar results are shown but in this case it is the displacement thickness $\delta_{1}$ that is kept constant to match that of the linear profile (that is $\delta_{1}=1 \%$ ). The exponent $n$ is varied and $\delta$ is adjusted to maintain $\delta_{1}$ constant. In this case there is again no visible impact of $n$ on the downstream modes but the impact on modes propagating upstream is significantly smaller than in figures $15 \mathrm{a}, \mathrm{c}$ (for instance of the order of $1 \mathrm{~dB}$ for $\xi \simeq 0.6$ ).

Calculations were also performed for other flow velocities, frequencies and liner resistance and the same conclusions are obtained. 

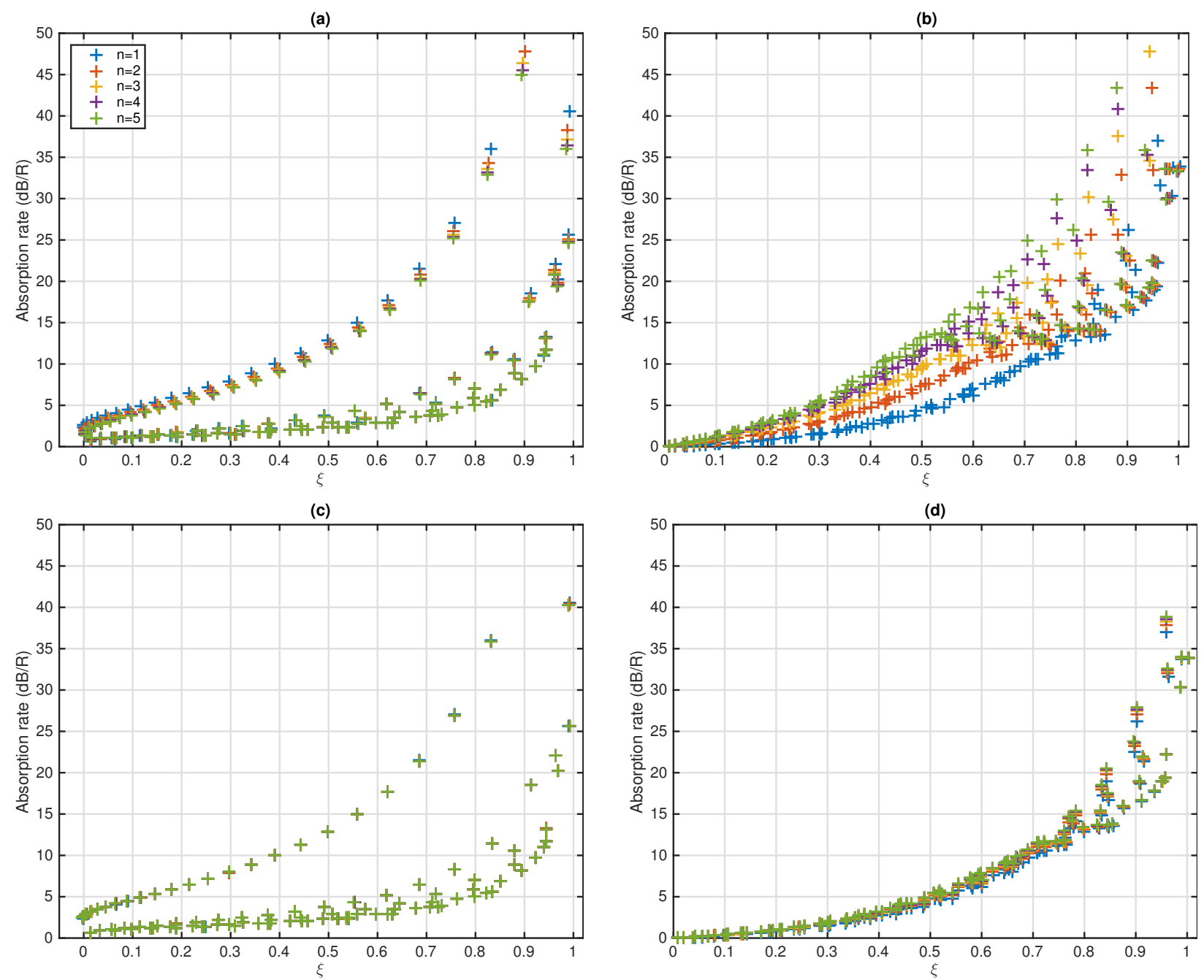

Figure 15: Absorption rate of the downstream modes (a,c) and upstream modes (b,d) as a function of $\xi$ for $M=0.5, \omega=23$ for the liner model (15) with $R_{\mathrm{L}}=4$. Fixed boundary layer thickness $\delta=2 \%(\mathrm{a}, \mathrm{b})$ or fixed displacement thickness $\delta_{1}=1 \%$ $(\mathrm{c}, \mathrm{d})$. 


\section{Conclusions and perspectives}

The effect of the mean flow boundary layer on liner performance was studied by calculating acoustic modes in a lined duct with flow. The parametric study assessed the effects of the flow velocity, liner resistance, frequency and boundary layer thickness and profile. These parameters were selected to be representative of liners found on current designs of turbofan engines. Results show that there is a complex interplay between the boundary layer thickness, the direction of propagation and the liner impedance. The boundary layer can have a strong impact on liner performance for typical configurations found on turbofan engines. The parametric study presented here clarifies the extent of this effect and the situations when it is significant.

For sound propagating downstream (e.g. in a bypass duct), the impact of the boundary layer is very limited and is only significant for a subset of modes (primarily the first radial modes which are behaving as surface waves). These modes are relatively well attenuated, and a difference of the order of $5 \mathrm{~dB}$ was observed at $1 \mathrm{BPF}$ between an infinitely thin boundary layer (Myers condition) and a fully-resolved boundary layer with $\delta_{1}=1 \%$. The attenuation of the other modes is generaly weaker and only slightly modified by the boundary layer. As a consequence, the attenuation of broadband noise propagating downstream is not very sensitive to the boundary layer.

For sound propagating upstream (e.g. intake liners), the effect of the boundary layer can be very significant (provided $M>0.3$ ). Unlike for downstream propagation, all the modes are influenced in a similar way, with the effect increasing with $\xi$. It can be large even for relatively well cut-on modes. For example, at $1 \mathrm{BPF}$ for the sideline configuration we observe a difference of $30 \mathrm{~dB}$ for the mode $(24,1)$ and more than $5 \mathrm{~dB}$ for most of the azimuthal orders. This leads to a significant impact on the attenuation of broadband noise, with a difference of the order of $5 \mathrm{~dB}$ observed in some cases between a fully-resolved boundary layer and the Myers condition.

The effect of the boundary layer depends strongly on the liner resistance, and the two cannot be separated. For upstream propagation, varying the liner resistance has shown a complete change in the trend associated with the boundary layer thickness. For a high liner resistance, a rapid reduction of the attenuation was observed when the boundary layer thickness increases while for a lower resistance, the change in attenuation is not as strong, and more complex. This indicates that interpreting the effect of the boundary layer purely in terms of sound refraction through the mean flow shear is too simplistic and that the interaction between the liner and the boundary layer is more complex. A parametric study where the impedance is progressively varied would provide additional information on the boundary layer effect, in particular if it changes significantly when the liner impedance is close to, or far from, the optimal impedance.

It was confirmed that it is recommended to use the displacement thickness to characterise the boundary layer. This makes the acoustic attenuation largely independent of the boundary layer profile.

We have shown how the modified impedance condition (6) can be implemented in the calculation of duct modes through the introduction of auxiliary variables. This impedance condition appears to be quite robust and in almost all cases there is a qualitative improvement over the Myers condition and the correct trend is captured. In some cases (typically a large liner resistance) the agreement with the exact solution is excellent. In some cases however it can still introduce noticeable levels of errors compared to predictions with a fully resolved boundary layer.

Based on the results for the present parametric study it appears that the use of a modified impedance condition should be recommended for predicting boundary layer effect on liner performance (and avoiding the inclusion of a fully-resolved boundary layer, which can be costly). However, its implementation in more general prediction methods such as finite element models will not be trivial due to the presence of high-order derivatives. In addition the behaviour of the acoustic and hydrodynamic fields close to impedance discontinuities between lined and hard surfaces will have to be better understood. Obtaining reliable information on the boundary layer along the intake liner will be necessary. The ability of RANS calculations to provide this information should be investigated, together with the use of experimental data. Other issues worth considering in this context are the differences between rig-scale and full-scale models, or between flight and static tests. 


\section{Acknowledgements}

Part of this work was funded by Innovate UK (HARMONY Programme, GA No. 101367) and the Rolls-Royce University Technology Centre on Gas Turbine Noise at the University of Southampton. The information and views set out in this paper are those of the author and do not necessarily reflect the official opinion of Rolls-Royce plc or Innovate UK. This work greatly benefited from discussions with the acoustic team at Airbus Toulouse as well as Christoph Richter (Rolls-Royce Deutschland) and Paul Murray. The author is also grateful to Alan McAlpine and Ed Brambley for providing verification data. The author acknowledges the use of the IRIDIS High Performance Computing Facility, and associated support services at the University of Southampton, in the completion of this work.

\section{References}

[1] M. Myers, On the acoustic boundary condition in the presence of flow, Journal of Sound and Vibration 71 (3) (1980) 429-434.

[2] E. Brambley, Fundamental problems with the model of uniform flow over acoustic linings, Journal of Sound and Vibration 322 (2009) 1026-1037.

[3] G. Gabard, E. Brambley, A full discrete dispersion analysis of time-domain simulations of acoustic liners with flow, Journal of Computational Physics 273 (2014) 310-326. doi:10.1016/j.jcp.2014.05.004.

[4] S. Rienstra, M. Darau, Boundary-layer thickness effects of the hydrodynamic instability along an impedance wall, Journal of Fluid Mechanics 671 (2011) 559-573.

[5] D. Marx, A piecewise linear mean flow model for studying stability in a lined channel, Journal of Sound and Vibration 331 (2012) 3809-3823.

[6] P. Drazin, W. Reid, Hydrodynamic stability, Cambridge University Press, 2004.

[7] W. Eversman, Effect of boundary layer on the transmission and attenuation of sound in an acoustically treated circular duct, Journal of the Acoustical Society of America 49 (5) (1971) 1372-1380.

[8] A. Nayfeh, J. Kaiser, B. Shaker, Effect of mean-velocity profile shapes on sound transmission through two-dimensional ducts, Journal of Sound and Vibration 34 (1974) 413-423.

[9] A. McAlpine, M. Fisher, B. Tester, "Buzz-saw" noise: A comparison of modal measurements with an improved prediction method, Journal of Sound and Vibration 306 (3) (2007) 419-443.

[10] Y. Renou, Y. Aurégan, Failure of the Ingard-Myers boundary condition for a lined duct: An experimental investigation, Journal of the Acoustical Society of America 130 (1) (2012) 52-60

[11] E. J. Rice, Spinning mode sound propagation in ducts with acoustic treatment and sheared flow, Technical Memorandum NASA TM X-71672, NASA, Lewis Research Center; Cleveland, OH, United States (1975).

[12] E. J. Rice, Attenuation of sound in ducts with acoustic treatment - A generalized approximate equation, Technical Memorandum NASA TM X-71830, NASA, Lewis Research Center; Cleveland, OH, United States (1975).

[13] E. J. Rice, Acoustic liner optimum impedance for spinning modes with mode cut-off ratio as the design criterion, Technical Memorandum NASA TM X-73411, NASA, Lewis Research Center; Cleveland, OH, United States (1976).

[14] E. J. Rice, Optimum wall impedance for spinning modes-a correlation with mode cutoff ratio, Journal of Aircraft 16 (5) (1979) 336-343.

[15] M. Myers, S. Chuang, Uniform asymptotic approximations for duct acoustic modes in a thin boundary-layer flow, AIAA Journal 22 (9) (1983) 1234-1241.

[16] Y. Aurégan, R. Starobinski, V. Pagneux, Influence of grazing flow and dissipation effects on the acoustic boundary conditions at a lined wall, Journal of the Acoustical Society of America 109 (1) (2001) 59-64.

[17] E. Brambley, Well-posed boundary condition for acoustic liners in straight ducts with flow, AIAA Journal 49 (6) (2011) $1272-1282$.

[18] G. Gabard, A comparison of impedance boundary conditions for flow acoustics, Journal of Sound and Vibration 332 (2013) $714-724$.

[19] M. Goldstein, An exact form of Lilley's equation with a velocity quadrupole/temperature dipole source term, Journal of Fluid Mechanics 443 (2001) 231-236.

[20] K. Ingard, Influence of fluid motion past a plane boundary on sound reflection, absorption and transmission, Journal of the Acoustical Society of America 31 (7) (1959) 1035-1036.

[21] W. Eversman, R. Beckemeyer, Transmission of sound in ducts with thin shear layers - convergence to the uniform flow case, Journal of the Acoustical Society of America 52 (1) (1972) 216-220.

[22] C. Tam, J. Webb, Dispersion-relation-preserving finite difference schemes for computational acoustics, Journal of Computational Physics 107 (1993) 262-281.

[23] J. Berland, C. Bogey, O. Marsden, C. Bailly, High-order, low dispersive and low dissipative explicit schemes for multiplescale and boundary problems, Journal of Computational Physics 224 (2007) 637-662.

[24] K. Mohseni, T. Colonius, Numerical treatment of polar coordinate singularities, Journal of Computational Physics 157 (2000) 787-795.

[25] J. P. Boyd, Chebyshev and Fourier spectral methods, Courier Dover Publications, 2001. 
[26] H. R. Lewis, P. M. Bellan, Physical constraints on the coefficients of Fourier expansions in cylindrical coordinates, Journal of Mathematical Physics 31 (11) (1990) 2592-2596. doi:10.1063/1.529009.

URL http://scitation.aip.org/content/aip/journal/jmp/31/11/10.1063/1.529009

[27] S. Rienstra, A classification of duct modes based on surface waves, Wave Motion 37 (2003) 119-135.

[28] E. Brambley, N. Peake, Classification of aeroacoustically relevant surface modes in cylindrical lined ducts, Wave Motion 43 (2006) 301-310.

[29] A. McAlpine, personal communication.

[30] R. Motsinger, R. Kraft, Design and performance of duct acoustic treatment, in: H. Hubbard (Ed.), Aeroacoustics of Flight Vehicles: Theory and Practice. Volume 2: Noise Control, 1991, pp. 165-206. 


\section{List of Figures}

1 Non-dimensional liner reactance $X_{\mathrm{L}}(\omega)$ as a function of the Helmholtz number $\omega$. . . . . .

2 Absorption rate as a function of $\xi$ for the modes propagating upstream with $M=0.5, \omega=23$ and the liner model (15) with $R_{\mathrm{L}}=4$ corresponding to $Z \simeq 4-\mathrm{i}\left(m_{\max }=28\right)$. Boundary layer thickness $\delta_{1}=0$ (black), $0.5 \%$ (blue), $1 \%$ (red), $1.5 \%$ (green). Fully-resolved boundary layer $(\cdot)$, Myers impedance condition $(\times)$ and modified impedance condition $(6)(+)$. . . . .

3 Modes propagating upstream calculated using the Myers condition for $M=0.5, \omega=23$ and the liner model (15) with $R_{\mathrm{L}}=4$. (a) Axial wavenumbers $k_{z}$ with colors representing $\operatorname{Im}\left(k_{r}\right)$. (b) Radial wavenumbers $k_{r}$ with colors representing $\operatorname{Im}\left(k_{z}\right) . \ldots \ldots \ldots \ldots \ldots$

4 Absorption rate as a function of $\xi$ for the modes propagating upstream with $M=0.5, \omega=23$ and an impedance $Z=2-\mathrm{i}\left(m_{\max }=28\right)$. Boundary layer thickness $\delta_{1}=0$ (black), $0.5 \%$ (blue), $1 \%$ (red), $1.5 \%$ (green). Fully-resolved boundary layer (.), Myers impedance condition $(\times)$ and modified impedance condition $(6)(+)$.

Modal attenuation as a function of the azimuthal order and radial order for the sideline case ( $\omega=28$ and $M=0.55$ ) with $\delta_{1}=1 \%$ and the liner model (15) corresponding to $Z \simeq 5-0.56 \mathrm{i}$. First (o), second $(\square)$ and third $(\diamond)$ radial modes. (a) Absorption rate in $\mathrm{dB} / \mathrm{R}$ predicted with the Myers condition (4) (blue), modified impedance condition (6) (red) and the fully-resolved boundary layer (black). (b) Difference between the predicted and exact absorption rate in

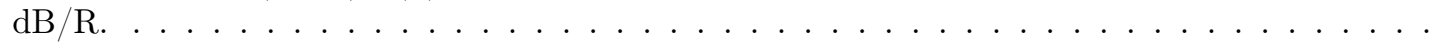

6 Absorption rate in $\mathrm{dB} / \mathrm{R}$ as a function of the boundary layer thickness for the first radial mode with $m=24$ for the sideline $(\omega=28$ and $M=0.55)$ case with the liner model (15)

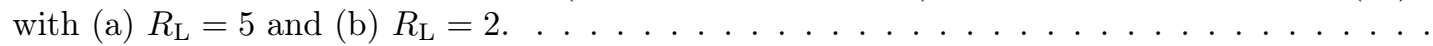

$7 \quad$ Mode shape function of the first radial mode with $m=24$ for the sideline case with the liner model (15) with (a) $R_{\mathrm{L}}=5$ and (b) $R_{\mathrm{L}}=2$. Myers condition: solid black line. Fully-resolved boundary layer: solid color lines. Modified impedance condition: dashed lines. . . . . . . . . .

8 Absorption rate as a function of $\xi$ for the modes propagating downstream with $M=0.5$, $\omega=23$ with the liner model (15) with (a) $R_{\mathrm{L}}=1$ or (b) $R_{\mathrm{L}}=2$. Boundary layer thickness $\delta_{1}=0$ (black), $0.5 \%$ (blue), $1 \%$ (red), $1.5 \%$ (green). Fully-resolved boundary layer (.), Myers impedance condition $(x)$ and modified impedance condition $(6)(+) . \ldots \ldots \ldots . . .$.

9 Modes propagating upstream calculated using a fully resolved boundary layer for $\delta_{1}=0.5 \%$, $M=0.5, \omega=23$ and the liner model (15) with $R_{\mathrm{L}}=1$. (a) Axial wavenumbers $k_{z}$ with colors representing $\operatorname{Im}\left(k_{r}\right)$. (b) Radial wavenumbers $k_{r}$ with colors representing $-\operatorname{Im}\left(k_{z}\right)$. . .

10 (a) Absorption rate in $\mathrm{dB} / \mathrm{R}$ as a function of the azimuthal order. (b) Difference between the impedance conditions and the fully-resolved boundary layer. Blue: Myers condition (4). Red: modified impedance condition (6). Black: fully-resolved boundary layer. First (o), second $(\square)$ and third $(\diamond)$ radial modes. Cutback configuration $(\omega=23$ and $M=0.5)$ with $\delta_{1}=1 \%$ and the liner model $(15)$ with $R_{\mathrm{L}}=1 \ldots \ldots \ldots \ldots \ldots$

11 Absorption rate in $\mathrm{dB} / \mathrm{R}$ as a function of the boundary layer thickness for the first radial mode with $m=24$ for the cutback case $(\omega=23$ and $M=0.5)$ with the liner model (15) with

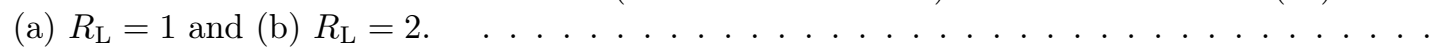

12 Attenuation of the upstream modes for the cutback condition $(\omega=23)$ with $R_{\mathrm{L}}=4$ and $\delta_{1}=1 \%$. Same notation as in figure $8 . \ldots \ldots \ldots \ldots \ldots$

13 Broadband attenuation rate as a function of frequency $\omega$ for the modes propagating downstream $(\mathrm{a}, \mathrm{c})$ and upstream $(\mathrm{b}, \mathrm{d})$. Cutback condition $(M=0.5)$ with the liner model $(15)$ with $R_{\mathrm{L}}=4(\mathrm{a}, \mathrm{b})$ and $R_{\mathrm{L}}=1(\mathrm{c}, \mathrm{d})$. Dot-dashed line: Myers condition (4). Solid line: Fully resolved boundary layer. Dashed line: Modified impedance condition (6). Boundary layer

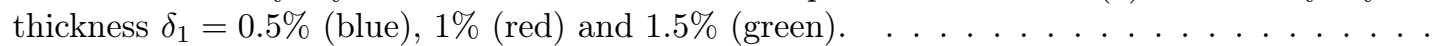

14 Boundary layer profiles (18) for a range of orders $n \ldots \ldots \ldots \ldots \ldots \ldots$

15 Absorption rate of the downstream modes (a,c) and upstream modes (b,d) as a function of $\xi$ for $M=0.5, \omega=23$ for the liner model (15) with $R_{\mathrm{L}}=4$. Fixed boundary layer thickness $\delta=2 \%(\mathrm{a}, \mathrm{b})$ or fixed displacement thickness $\delta_{1}=1 \%(\mathrm{c}, \mathrm{d}) \ldots \ldots \ldots \ldots \ldots$ 